\title{
IL-33 targeting attenuates intestinal mucositis and enhances effective tumor chemotherapy in mice
}

\author{
R Guabiraba ${ }^{1}$, AG Besnard ${ }^{1}$, GB Menezes ${ }^{2}$, T Secher ${ }^{3}$, MS Jabir ${ }^{1,4}$, SS Amaral ${ }^{2}, \mathrm{H} \mathrm{Braun}^{5,6}$, \\ RCP Lima-Junior ${ }^{7}$, RA Ribeiro ${ }^{7}$, FQ Cunha ${ }^{8}$, MM Teixeira ${ }^{9}$, R Beyaert ${ }^{5,6}$, GJ Graham ${ }^{1}$ and FY Liew ${ }^{1,10}$
}

Intestinal damage and severe diarrhea are serious side effects of cancer chemotherapy and constrain the usage of most such therapies. Here we show that interleukin-33 (IL-33) mediates the severe intestinal mucositis in mice treated with irinotecan (CPT-11), a commonly used cancer chemotherapeutic agent. Systemic CPT-11 administration led to severe mucosal damage, diarrhea, and body weight loss concomitant with the induction of IL-33 in the small intestine (SI). This mucositis was markedly reduced in mice deficient in the IL-33R $\left(\mathrm{ST}^{-/-}\right)$. Moreover, recombinant IL-33 exacerbated the CPT-11-induced mucositis, whereas IL-33 blockade with anti-IL-33 antibody or soluble ST2 markedly attenuated the disease. CPT-11 treatment increased neutrophil accumulation in the SI and adhesion to mesenteric veins. Supernatants from SI explants treated with CPT-11 enhanced transmigration of neutrophils in vitro in an IL-33-, CXCL1/2-, and CXCR2dependent manner. Importantly, IL-33 blockade reduced mucositis and enabled prolonged CPT-11 treatment of ectopic CT26 colon carcinoma, leading to a beneficial outcome of the chemotherapy. These results suggest that inhibition of the IL-33/ST2 pathway may represent a novel approach to limit mucositis and thus improve the effectiveness of chemotherapy.

\section{INTRODUCTION}

Mucositis is defined by inflammatory and ulcerative lesions of the oral and gastrointestinal mucosa commonly associated with cancer chemotherapy. ${ }^{1,2}$ Combination therapy such as radiation with concurrent chemotherapy may further increase the severity of mucositis that often leads to dosage reduction or premature cessation of cancer treatment. ${ }^{3,4}$ Thus, reagents that can attenuate chemotherapy-induced mucositis would be highly beneficial in enabling prolonged therapy and hence more effective cancer treatment.

Mucositis develops as a consequence of epithelial injury. ${ }^{2}$ However, its physiopathology is complex and involves multiple steps ${ }^{1}$ including the generation of reactive oxygen species and reactive nitrogen species, together leading to epithelial damages. ${ }^{5}$ Chemotherapy directly causes DNA damage and cell death ${ }^{6}$ with activation of nuclear factor- $\kappa B$ and upregulation of cytokine production..$^{7-10}$ In the ulcerative phase, epithelial erosion can lead to risk of microbial infiltration and septic shock. $^{11}$

CPT-11, a topoisomerase I inhibitor, is an antiproliferative drug used to treat different types of human malignancies, such as metastatic colorectal cancer. ${ }^{3,4} \mathrm{CPT}-11$ is metabolized in the liver and converted to $\mathrm{SN}-38$, the active metabolite, by carboxylesterase-mediated hydrolysis. ${ }^{3}$ The intestinal microbiota enzymatic system is also involved in the metabolism of CPT-11, and the compound can be metabolized in different in vitro and ex vivo experimental settings. ${ }^{3,12-14}$ The clinical pharmacokinetic properties of CPT-11 and its metabolites appear to be crucial for optimal anticancer chemotherapy. ${ }^{3}$

\footnotetext{
${ }^{1}$ Institute of Infection, Immunity and Inflammation, Glasgow Biomedical Research Centre, University of Glasgow, Glasgow, UK. ${ }^{2}$ Immunobiophotonics, Department of Morphology, Biological Sciences Institute, Federal University of Minas Gerais, Belo Horizonte, Minas Gerais, Brazil. ${ }^{3}$ Centre de Physiopathologie de Toulouse Purpan, U1043, Institut National de la Santé et de la Recherche Médicale, Toulouse, France. ${ }^{4}$ Departmet of Biotechnology, University of Technology, Baghdad, Iraq. ${ }^{5}$ Department of Biomedical Molecular Biology, Ghent University, Ghent, Belgium. ${ }^{6} \mathrm{VIB}$ Inflammation Research Center, Ghent, Belgium. ${ }^{7}$ Department of Physiology and Pharmacology, Faculty of Medicine, Federal University of Ceará, Ceará, Brazil. ${ }^{8}$ Department of Pharmacology, School of Medicine of Ribeirão Preto (FMRP), University of São Paulo, São Paulo, Brazil. ${ }^{9}$ Immunopharmacology, Department of Biochemistry and Immunology, Biological Sciences Institute, Federal University of Minas Gerais, Belo Horizonte, Minas Gerais, Brazil and ${ }^{10} \mathrm{CEGMR}$, King Abdulaziz University, Jeddah, Saudi Arabia. Correspondence: FY Liew or R Guabiraba (foo.liew@glasgow.ac.uk or rguabiraba@gmail.com)
}

Received 18 October 2013; accepted 16 December 2013; published online 15 January 2014. doi:10.1038/mi.2013.124 
Interleukin (IL)-33 is a member of the IL- 1 cytokine family that also includes IL-1 $\beta$ and IL-18 ( see ref. 15). IL-33 is crucial for the induction of type 2 immune responses by promoting the synthesis of cytokines such as IL- 5 and IL- 13 by T helper type 2 (Th2) lymphocytes, mast cells, basophils, and eosinophils. IL-33 is also involved in the induction of non-Th2-type acute and chronic inflammation as a proinflammatory cytokine. ${ }^{15,16}$ IL-33 signals via a heteromeric receptor that consists of ST2 and IL-1R accessory protein. ${ }^{17}$ ST2 (also known as T1), the transmembrane protein encoded by the ST2 gene, is expressed especially on immune cells such as mast cells and activated Th2 cells. ${ }^{18,19}$ The ST2 gene is alternatively spliced to produce a soluble form (sST2) that acts as an IL-33 decoy receptor. ${ }^{20}$ IL-33 is produced as a precursor protein (pro-IL-33) that is proteolytically converted to mature IL-33. Both forms are released by necrotic cells and have biological activity. ${ }^{19-21}$ Thus, IL-33 released by necrotic cells during tissue injury may play a damage-associated molecular pattern/alarmin-like role in the induction of inflammation. ${ }^{16}$

IL-33 is expressed by the gut epithelial cells, ${ }^{15}$ but current data on the role of IL-33 in the onset of inflammatory bowel diseases (IBD) are controversial. ${ }^{22}$ IL-33 appears to enhance intestinal inflammation in disease models driven by Th2 and innate immune responses, such as in senescence-acceleratedprone mice (SAMP) and experimental acute colitis, and possibly in ulcerative colitis (UC) patients. ${ }^{23-27}$ Upregulation of IL-33 in patients with IBD has been demonstrated by several reports (reviewed in). However, the participation of IL-33 in patients undergoing chemotherapy treatment has so far not been documented. High levels of IL-33 during acute inflammation are likely to exacerbate tissue damage, whereas they may enhance tissue repair during recovery. ${ }^{22,26}$ Thus, the initial features of the specific immune response and the timing of IL-33 blockade may define the disease outcome.

Animal models of CPT-11-induced mucositis are used extensively to identify the key players in disease pathogenesis, such as cytokines and chemokines. ${ }^{10,12,28-30}$ However, the sequence of events following mucosal damage induced by chemotherapy remains undefined. Here we report a hitherto unrecognized mechanism by which IL-33 mediates CPT-11induced mucositis via the attraction of neutrophils to the site of inflammation and tissue damage in the small intestine (SI). Targeting the IL-33/ST2 pathway confers protection and tissue preservation. In a murine model of CT26 ectopic colon carcinoma, IL-33 blockade enables prolonged and effective chemotherapy, resulting in markedly reduced tumor growth. These results suggest that the IL-33/ST2 pathway might be a novel therapeutic target for an enhanced beneficial outcome of cancer chemotherapy.

\section{RESULTS}

IL-33 is produced in chemotherapy-induced mucositis in the SI

Several proinflammatory cytokines have been associated with the severity of chemotherapy-induced mucositis, although their role in disease pathogenesis is not fully understood. ${ }^{1,2,10,31}$ To investigate the role of IL-33 in the inflammatory response induced by CPT-11 (irinotecan) chemotherapy, BALB/c mice were injected intraperitoneally (i.p.) for 4 consecutive days (0 to 3) with CPT-11 and mucositis was analyzed on day 4 . The production of IL-33 and its soluble receptor sST2 was significantly elevated in the SI as compared with that of the phosphate-buffered saline (PBS)-treated control mice (Figure 1a). The production of IL-33 and sST2 appears to be confined to the mucosal compartment as they were not detected in the serum (data not shown). We then verified this observation using SI explant of naive mouse in vitro. CPT11 stimulated IL-33 production by the SI explant in a dosedependent manner (Figure 1b). Furthermore, SN-38, the active metabolite of CPT-11, also induced IL-33 synthesis by the SI explant in vitro (Figure 1b).

To investigate the cellular source of IL-33, the SI of CPT-11treated mice was analyzed by immunohistochemical (IHC) staining. IL- $33^{+}$cells were markedly increased in the epithelial cells of the villi and the crypts of the CPT-11-treated mice compared with that of the PBS-treated mice (Figure 1c). Positive staining for IL-33 was seen in the whole epithelia, suggesting that both putative stem cells/proliferating progenitor cells (crypts) and differentiated cells (villi, including goblet cells, enterocytes, and enteroendocrine cells) could release IL-33. IHC staining using Annexin V, which binds to phosphatidylserine to identify apoptotic cells, confirmed that both differentiated and proliferating epithelial cells were damaged by CPT-11 and are potential sources of IL-33 (Figure 1c). To confirm this observation, we then isolated the epithelial cells $\left(\mathrm{CD} 45^{-}\right.$cytokeratin $\left.^{+}\right)$from the SI of naive mice and cultured the cells for $6 \mathrm{~h}$ with CPT- 11 in vitro and analyzed the expression of IL- 33 by fluorescence-activated cell sorting. CPT11-stimulated epithelial cells produce IL-33 in a concentrationdependent manner (Figure 1d,e).

We then examined the relevance of our observation in human cells using the epithelial-like human colon carcinoma Caco- 2 cells. Caco- 2 cells produced significant amounts of IL33 when cultured in vitro with CPT-11 and SN-38 (Figure 1f). There was also a modest but significant reduction in cell viability of Caco- 2 cells at this time point (10\% for CPT- 11 and $15 \%$ for SN-38 as compared with control group). Together, these results demonstrate that CPT-11 effectively induces IL-33 production by the epithelial cells of the SI.

\section{IL-33 is associated with the pathogenesis of chemotherapy- induced mucositis}

We next investigated the role of the IL-33/ST2 pathway in the pathogenesis of mucositis. We treated wild-type (WT) BALB/c mice and $\mathrm{ST} 2^{-1-}$ mice (BALB/c background) with CPT-11 with or without recombinant murine IL-33. WT mice treated with CPT-11 showed significant body weight loss $(\sim 10 \%)$ by day 3 and diarrhea (clinical score 1-2) on day 4 (Figure 2a,b). These clinical changes were accompanied by severe systemic leucopenia, shortened villi, and increased plasma leakage in the SI (Figure 2c-e). All these parameters were significantly 

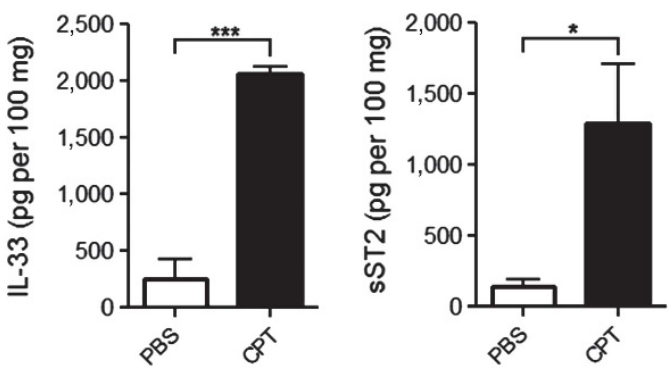

c
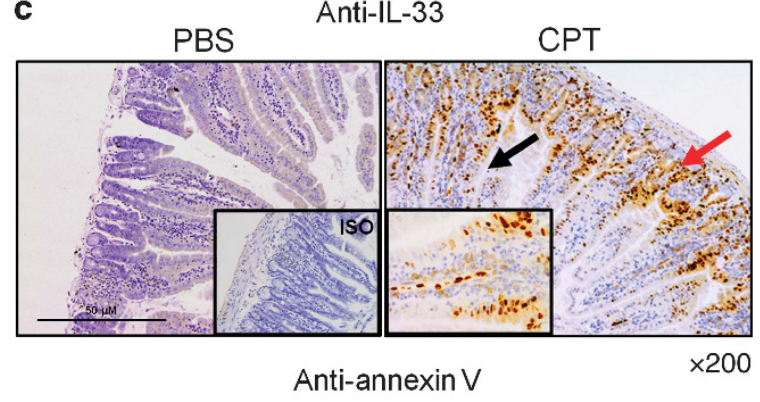

$\times 200$

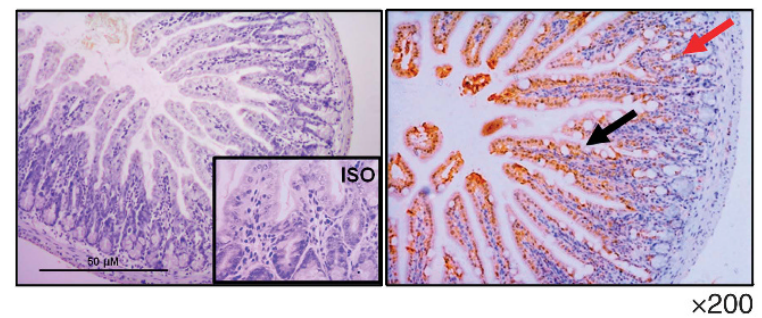

e

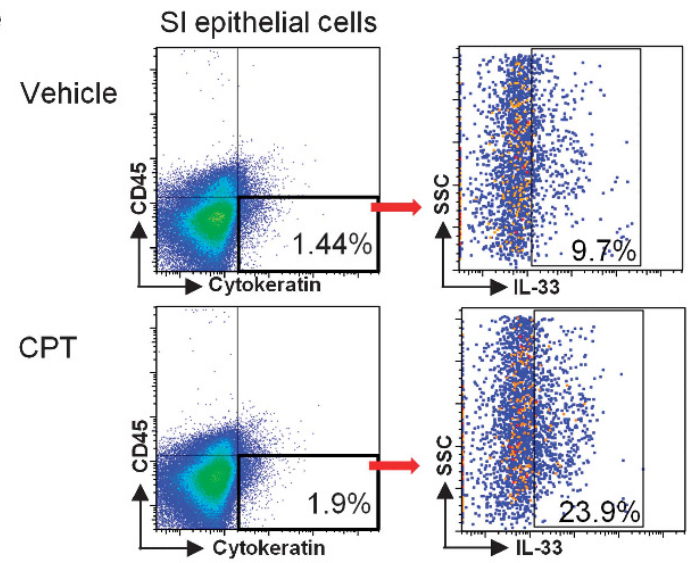

b Sl explant

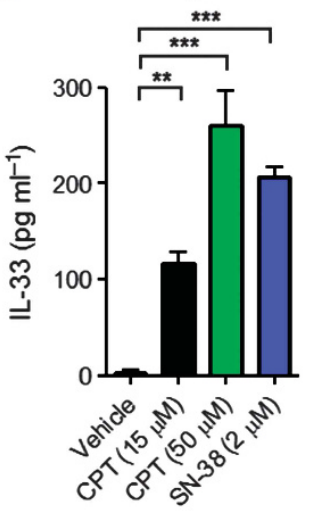

d SI epithelial cells

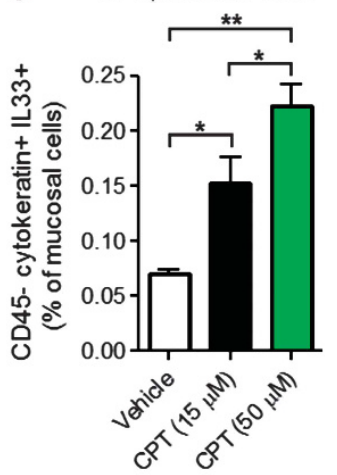

f

Caco-2

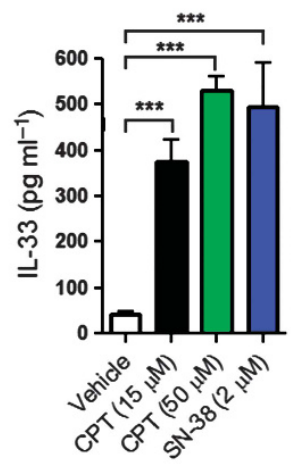

Figure 1 Interleukin-33 (IL-33) is produced in the small intestine (SI) in chemotherapy-induced mucositis. (a) IL-33 and soluble ST2 (sST2) concentrations in the SI of BALB/c mice after four daily injections of CPT-11 $\left(60 \mathrm{mg} / \mathrm{kg}^{-1}\right)$ or phosphate-buffered saline (PBS) were determined by enzyme-linked immunosorbent assay (ELISA). (b) IL-33 production in vitro by SI explants from BALB/c mice after $24 \mathrm{~h}$ of incubation with CPT-11 or SN-38 measured by ELISA. (c) Representative immunohistochemical (IHC) staining for IL-33 and Annexin V in the SI of PBS or CPT-11-treated mice at day 4. Original magnification $\times 200$. Arrows indicate positive staining for IL-33 and Annexin V in the villi (black) and crypts (red). Inserts: ISO: original magnification $\times 200$; CPT-11: original magnification $\times 400$. (d) The frequency of IL-33 ${ }^{+}$CD $45^{-}$cytokeratin ${ }^{+}$epithelial cells $(e p T s)$ from mice treated with CPT-11 or PBS (vehicle). (e) Representative fluorescence-activated cell sorting (FACS) analysis showing the gating of IL-33 ${ }^{+} \mathrm{CD}^{-} 5^{-} \mathrm{Cytokeratin}^{+}$ epTs isolated from wild-type (WT) mice treated with CPT-11 $(50 \mu \mathrm{M})$ for $6 \mathrm{~h}$. (f) IL-33 concentration in the supernatant of Caco-2 cells cultured for $48 \mathrm{~h}$ with CPT-11 or SN-38 determined by ELISA. Results are representative of two independent experiments $\left(n=3-5\right.$ mice per group). ${ }^{*} P<0.05,{ }^{\star \star} P<0.01$, and ${ }^{* * *} P<0.001$.

exacerbated by the additional daily treatment with exogenous IL-33 (200 ng per mouse). ST2 ${ }^{-l-}$ mice did not respond to recombinant murine IL-33 treatment in any of the clinical parameters evaluated (Figure 2a,b). Furthermore, ST2 $2^{-1-}$ mice developed significantly attenuated disease with reduced weight loss, clinical score (clinical score 0-1), leucopenia, 


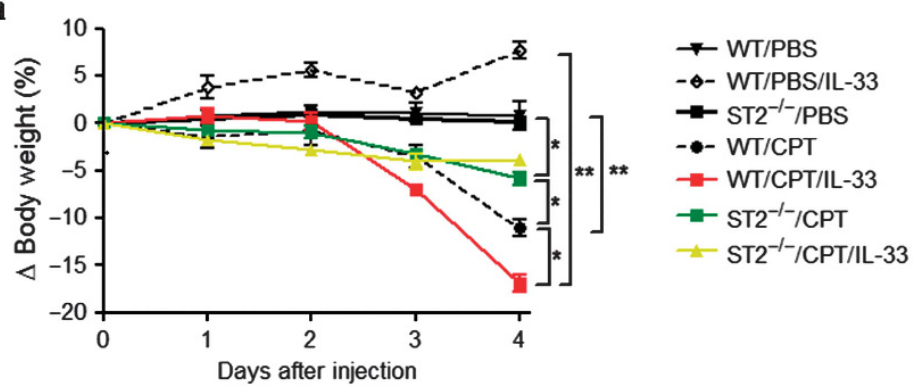

d
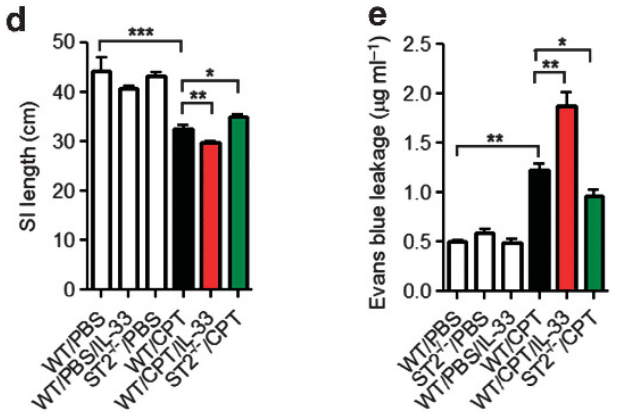

i

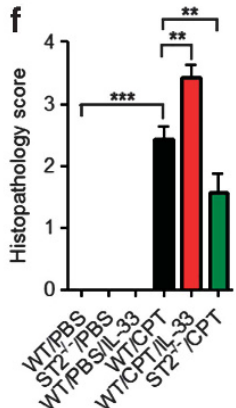

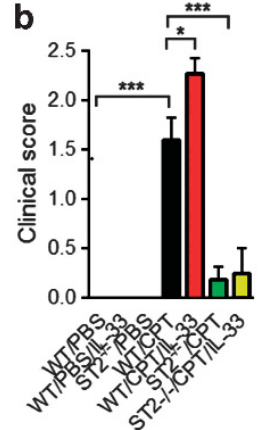

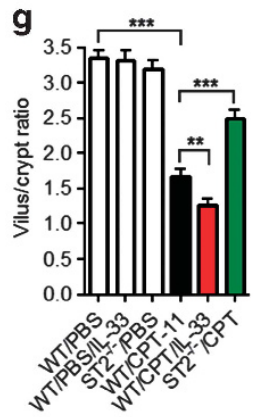

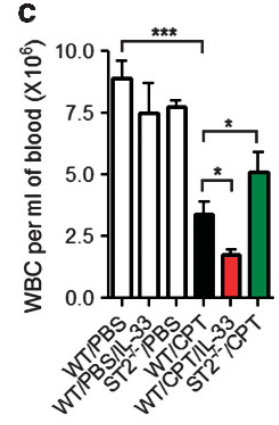

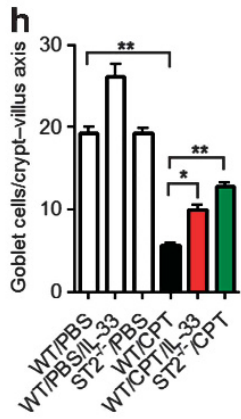

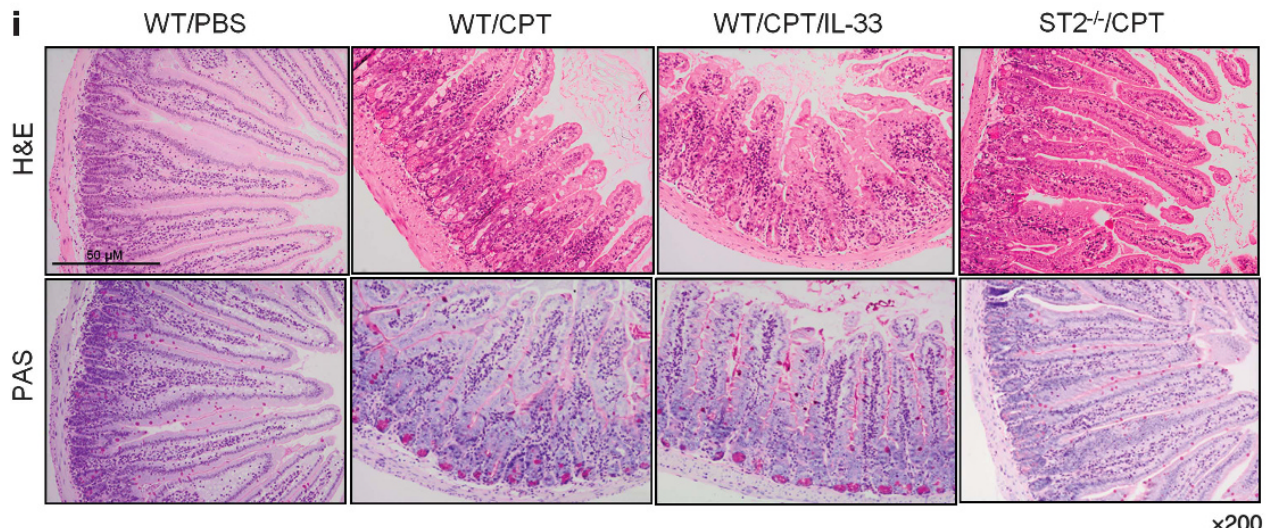

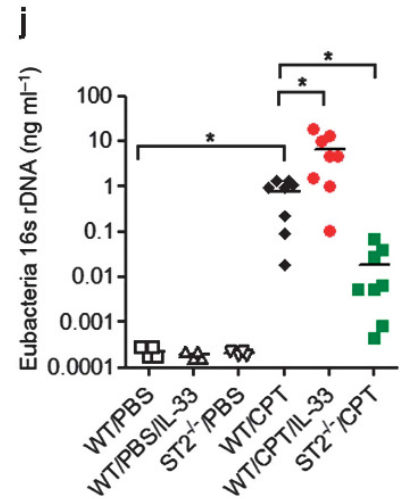

Figure 2 Interleukin-33 (IL-33) is associated with the pathogenesis of chemotherapy-induced mucositis. Wild-type (WT) and ST2 ${ }^{-/-}$mice were treated with CPT-11 or phosphate-buffered saline (PBS) with or without IL-33. (a) $\Delta$ Body weight loss (\%) was measured daily and (b) clinical score determined on day 4. (c) Whole blood cell (WBC) counts, (d) small intestine (SI) length, and (e) Evans blue leakage were also determined on day 5. (f) Histopathology score, (g) villus/crypt ratio, and (h) Goblet cell counts in the crypt/villus axis are also shown. (i) Representative histology of SI samples on day 5. (j) Expression of Eubacteria 16s rDNA in the blood was evaluated by quantitative polymerase chain reaction (qPCR) on day 4. Data are pool of two experiments ( $n=4-8$ mice per group) and are representative of three independent experiments. ${ }^{*} P<0.05,{ }^{* *} P<0.01$, and ${ }^{\star * *} P<0.001$.

plasma leakage, but increased SI villi length, as compared with the PBS control group (Figure 2a-e). Histopathological analysis of the SI of WT mice revealed a dramatic remodeling of the small intestinal mucosa, with different degrees of submucosal edema, submucosal hemorrhage, venous congestion, inflammatory submucosal leukocyte infiltrate, shortening of villi, and reduction in goblet cell numbers as assessed by hematoxylin-eosin and periodic acid-Schiff staining (Figure 2f-i). Mice treated with CPT-11+ IL-33 showed even more severe outcome in all these parameters and presented increased signs of hemorrhage, venous congestion, and villi reduction. A modest but significant deleterious effect of CPT-11 + IL-33 treatment in the mucosa starts to appear on day 3 in the WT mice (data not shown). In contrast, ST2 ${ }^{-1-}$ mice presented a preserved intestinal architecture, with subtle leukocyte infiltrate and discrete villi shortening. Villi atrophy and crypt destruction are the main causes of diarrhea in mice. $^{32-34}$

As mucosal damage due to chemotherapy has been associated with increased blood bacteremia, ${ }^{11,32,35}$ we also measured the level of bacteremia by quantitative polymerase chain reaction (qPCR). Mice treated with CPT-11 developed markedly increased bacteremia that was significantly elevated by cotreatment with IL-33 (clinical score 2-3), whereas ST2 ${ }^{-1-}$ mice treated with CPT-11 (clinical score $0-1$ ) had significantly less bacteremia compared with that of the WT mice (Figure 2j). 
Together, these results demonstrate that CPT-11-induced mucositis is dependent on the IL-33/ST2 pathway.

The role of chemokines in the CPT-11-induced mucositis The histopathological changes observed above were associated with a significant increase in the level of expression of the apoptosis markers Caspase 9, Caspase 3 and poly (ADP-ribose) polymerase (cleaved forms), and Bax in the SI (Supplementary Figure S1a-c online). IL-33 further increased the expression of these apoptotic markers that were significantly reduced in $\mathrm{ST} 2^{-1-}$ mice. We also investigated the production of selected chemokines in the serum (Supplementary Figure S1d-f) and in the SI (Supplementary Figure S1g-i). The concentrations of CXCL1/KC, CXCL2/MIP-2, and CCL2/JE in the serum were all elevated in the CPT-11-treated mice as compared with untreated mice. All these chemokines were further increased by the additional treatment with IL-33. In contrast, the levels of these chemokines remained at the control (PBS) level in the ST2 ${ }^{-1-}$ mice. Importantly, IL-33 treatment increased the level of IL-33 in the SI induced by CPT-11. This increase was not because of the injected recombinant murine IL-33, as the level of IL-33 in IL-33-treated WT mice not given CPT-11 remained low. Moreover, the SI was perfused before cytokine/chemokine assays (see Methods). ST2 ${ }^{-1-}$ mice treated with CPT-11 had significantly lower concentration of IL-33 in the SI compared with CPT-11-treated WT mice (Supplementary Figure 1j). These data suggest that IL-33-mediated chemokine induction may be an important, though not the only, mechanism involved in CPT-11-induced mucositis. In addition, IL-33 released by damaged epithelial cells (i.e., IL- $33^{+}$Annexin $\mathrm{V}^{+}$cells) could increase its own synthesis by activating the $\mathrm{ST} 2$ receptor in an autocrine manner. ${ }^{36}$ It has been shown that the local allergic inflammatory response is amplified by dendritic cell-produced IL-33 through potential autocrine regulation. ${ }^{37}$ In line with this observation, the ST2 receptor is known to be widely expressed in intestinal epithelial cells. ${ }^{25}$

\section{Neutrophils play a key role in CPT-11/IL-33-induced intestinal mucositis}

The marked increase of CXCL1 and CXCL2 during mucositis induced by the CPT-11/IL-33/ST2 pathway suggests that neutrophils might play an important role in the downstream events leading to mucosal damage. Although neutropenia and neutropenic fever are frequently reported in patients undergoing chemotherapy, the role of neutrophils in the context of CPT-11/IL-33-induced inflammatory response is unclear. ${ }^{38}$ Studies in rodents suggested that neutrophils may accumulate in the intestinal mucosa after chemotherapeutic treatment. ${ }^{9,10,39,40}$ We therefore examined the potential involvement of neutrophils in our system. WT and $\mathrm{ST} 2^{-1-}$ mice were treated as above and the presence of neutrophils in the SI was examined by myeloperoxidase (MPO) assay and IHC. CPT-11 treatment induced neutrophil accumulation in the SI from day 2 (data not shown) along the villi and increased progressively to day 5 in the WT mice but not in the ST2 ${ }^{-1-}$ mice (Figure 3a,b). The MPO activity in the SI of WT mice was further enhanced by the administration of IL-33
(Figure 3b). ST2 $2^{-1-}$ mice did not respond to IL-33 treatment in any of the parameters evaluated (data not shown).

We then characterized the neutrophils activated by CPT- 11 . Neutrophils $\left(\mathrm{CD} 11 \mathrm{~b}^{+} \mathrm{Ly}_{6 \mathrm{G}}{ }^{+}\right)$from WT mice were highly activated in the blood 3 days after CPT-11 treatment, a time point when IL-33 production in the SI was already increased $\left(600 \pm 13 \mathrm{pg} \mathrm{ml}^{-1}\right.$ compared with $42 \pm 7 \mathrm{pg} \mathrm{ml}^{-1}$ in $\mathrm{PBS}$ control) and the concentration of CXCL1/KC in the serum was elevated $\left(150 \pm 9 \mathrm{pg} \mathrm{ml}^{-1}\right.$ compared with $6 \pm 1.3$ in PBS control). These cells expressed the CXC chemokine receptor CXCR2 and CD62L (L-selectin) (Figure 3c-e). This time point preceded the severe neutropenia observed at later stages of the disease (data not shown). The neutrophils accumulated in the SI of the WT mice also showed highly activated phenotype expressing CXCR2 but shedding of CD62L (Figure 3f-h). Additional IL-33-treatment led to increased percentage and activation of neutrophils, with expression of CXCR2 and CD62L in the blood from CPT-11-treated mice (Figure 3e). $\mathrm{CD} 62 \mathrm{~L}$ expression was again reduced in the neutrophils from the SI of mice treated with CPT-11 + IL-33 compared with that in mice treated with CPT-11 alone (Figure $3 \mathbf{h}$ ). In contrast, little or no neutrophil activation was observed in the blood or SI of ST2 ${ }^{-1-}$ mice treated with CPT-11.

To further investigate the role of neutrophils in chemotherapy-induced intestinal mucositis, we first asked whether CPT-11 could induce the production of CXC chemokines in the SI. We cultured the SI explants from WT and ST2 ${ }^{-1-}$ mice with CPT-11, SN-38, or IL-33 and measured the production of CXCL1 and CXCL2 by enzyme-linked immunosorbent assay (ELISA). CPT-11 and SN-38 induced a significant amount of CXCL1 and CXCL2 in the SI explants from WT mice (Figure 3i,ji). The levels of chemokines produced by the SI of ST2 ${ }^{-1-}$ mice were significantly reduced compared with that of the WT mice. IL-33 alone induced a robust production of CXCL1 and CXCL2 in the SI of WT mice but not in the SI of ST2 ${ }^{-1-}$ mice (Figure $3 \mathbf{i}, \mathbf{j}$ ). These data suggest that although IL-33 is a potent inducer of CXCL1 and CXCL2 in the SI explants, CPT-11 can also induce a modest production of these chemokines independently of IL-33.

To determine the relevance of these findings in neutrophil migration, we assessed neutrophil recruitment in a transwell chamber in the presence of supernatants from SI explants treated with CPT-11 or SN-38 for $24 \mathrm{~h}$. Supernatants from WT SI explants treated with CPT-11 or SN-38 significantly induced the recruitment of neutrophils (Figure 3k). Supernatant from ST2 ${ }^{-1-}$ SI explants presented a $50-60 \%$ reduction in the effectiveness to induce neutrophil recruitment. Supernatant from WT (but not ST2 $2^{-1-}$ ) SI explants treated with IL-33 alone also induced the recruitment of neutrophils. To confirm the active role of IL-33 in the SI supernatants, we added sST2 to the supernatant in the transwell experiment. The IL-33 decoy receptor, sST2, significantly reduced neutrophil migration attracted by the supernatants from CPT-11- or SN-38-treated WT SI (Figure 31).

Together, these results demonstrate that neutrophils play a key role in the CPT-11-induced mucositis, at least partly, via the 
a

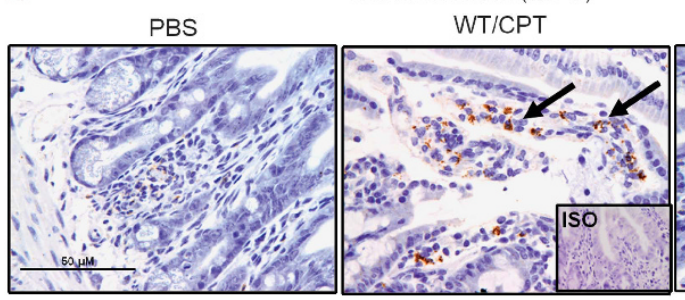

ST2 $2^{-/ /} \mathrm{CPT}$

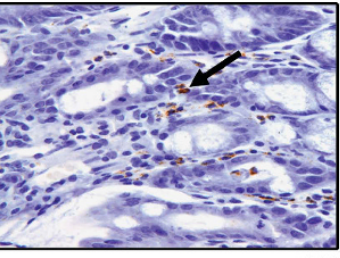

b

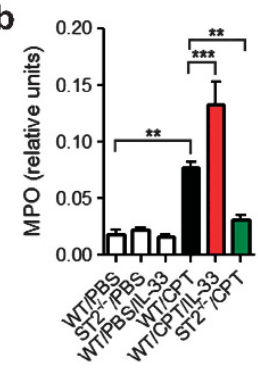

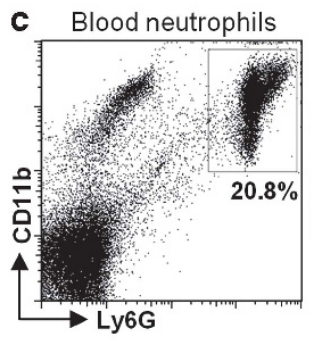
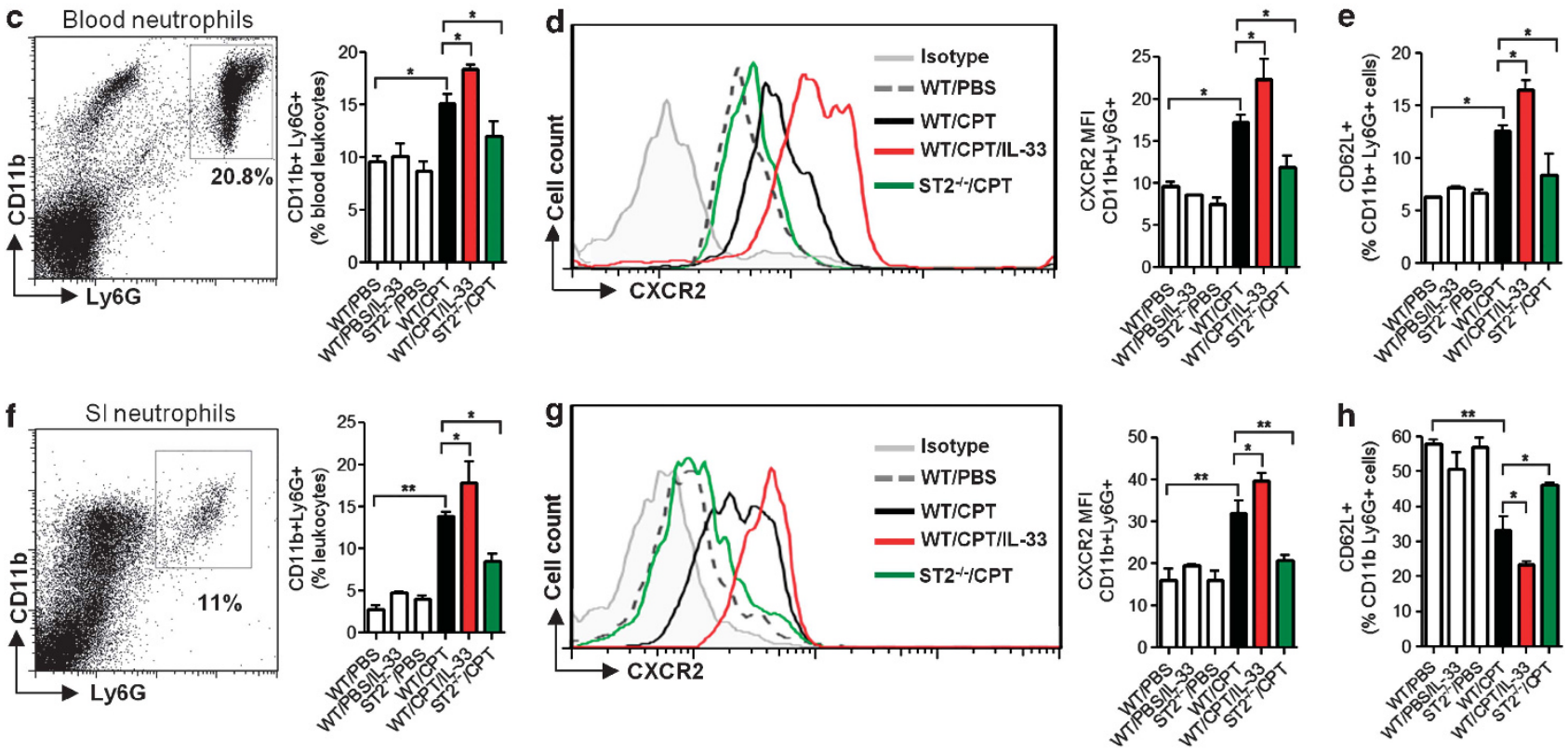

i

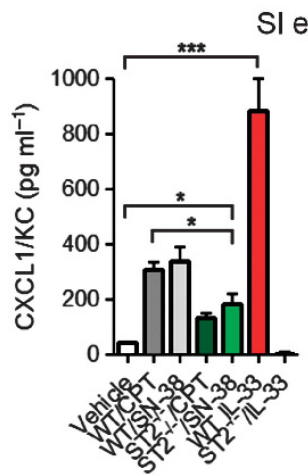

j

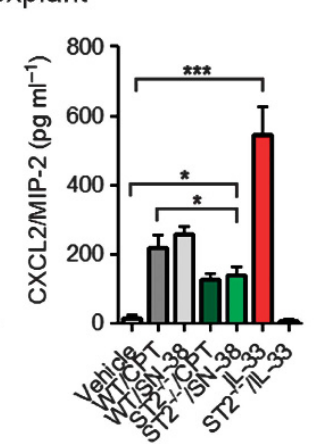

k

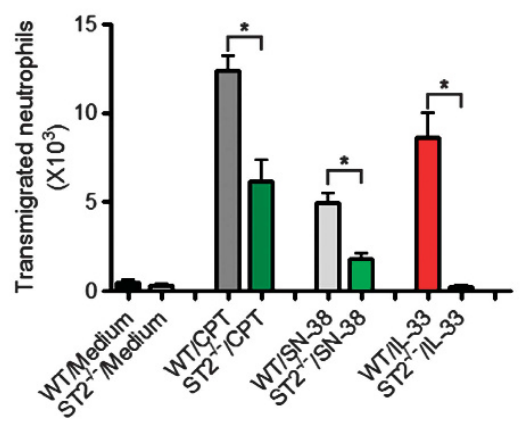

I

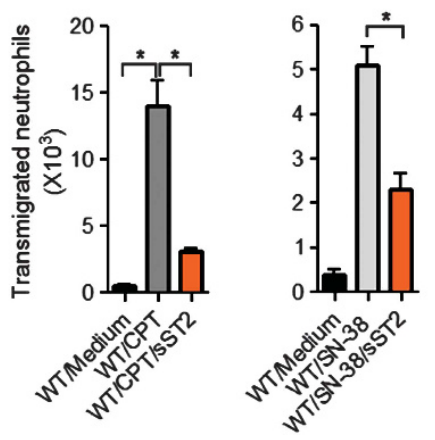

Figure 3 Neutrophils play a key role in CPT-11/interleukin-33 (IL-33)-induced intestinal mucositis. Wild-type (WT) and ST2 ${ }^{-1-}$ mice were treated with CPT-11 with or without IL-33. (a) Representative immunohistochemical (IHC) staining for myeloperoxidase (MPO) in the small intestine (SI) on day 2. Arrows indicate positive staining for MPO of the villi. (b) MPO activity in the SI on day 4. (c) Gating and representative percentage of blood CD11 ${ }^{+}$Ly $6 G^{+}$ neutrophils on day 2. (d) Representative mean fluorescence intensity (MFI) of CXCR2 on blood CD11b ${ }^{+}$Ly6G $^{+}$neutrophils on day 2. (e) Percentage of $\mathrm{CD} 2 \mathrm{~L}^{+} \mathrm{CD}_{11 \mathrm{~b}^{+}} \mathrm{Ly}_{6 \mathrm{G}}{ }^{+}$neutrophils in the blood on day 2. (f) Gating and representative percentage of CD11b ${ }^{+} \mathrm{Ly}_{6 \mathrm{G}}{ }^{+}$neutrophils in the SI on day 4 . (g) Representative MFI of CXCR2 on CD11 b ${ }^{+} \mathrm{Ly}_{6 G}{ }^{+}$neutrophils in the SI on day 4. (h) Percentage of CD62L ${ }^{+} \mathrm{CD} 11 \mathrm{~b}^{+} \mathrm{Ly}_{6 \mathrm{G}}{ }^{+}$cells in the SI on day 4. SI explants from WT and ST2 ${ }^{-1-}$ mice were cultured for $24 \mathrm{~h}$ in the presence of CPT-11 $(50 \mu \mathrm{m})$, SN-38 $(2 \mu \mathrm{m})$, or IL-33 (10 ng ml $\left.{ }^{-1}\right)$, and the concentrations of (i) CXCL1/KC and (j) CXCL2/MIP-2 determined by enzyme-linked immunosorbent assay (ELISA). (k, I) SI explants from WT or ST2 ${ }^{-1-}$ mice were cultured with CPT-11, SN-38, or IL-33. Supernatants were collected after $24 \mathrm{~h}$ and placed in the lower chamber of transwell culture plates. WT LyG6 ${ }^{+}$neutrophils were placed on the upper chamber. (k) The number of neutrophils in the lower chamber was determined $3 \mathrm{~h}$ later. (I) The number of transmigrating neutrophils in the presence of soluble ST2 (SST2; $10 \mu \mathrm{g} \mathrm{ml}^{-1}$ ) in the lower chamber is also shown. Results are representative of three independent experiments ( $n=5-6$ mice per group). ${ }^{*} P<0.05,{ }^{* *} P<0.01$, and ${ }^{* * *} P<0.001$. 
IL-33/ST2-mediated elevation of the chemokine production and chemokine receptor expression.

\section{IL-33 blockade ameliorates CPT-11-induced intestinal mucositis}

We next investigated whether the reduced mucositis in the ST2 ${ }^{-1-}$ mice can be reproduced by blocking endogenous IL-33 using an anti-IL-33 antibody and sST2. WT mice were treated with CPT-11 for 4 consecutive days with or without daily injection of anti-IL-33 or sST2. Both anti-IL-33 and sST2 significantly reduced body weight loss and clinical score compared with PBS-injected control mice (Figure 4a,b). The disease amelioration was accompanied by a reduction in tissue injury and signs of hemorrhage and congestion (Figure 4c), with reduced villi shortening (Figure 4d). Neutrophil accumulation and the production of CXC and CC chemokines CXCL1, CXCL2, and CCL2 in the SI were also significantly reduced in the anti-IL33- and sST2-treated mice compared with PBS-treated control mice (Figure $4 \mathbf{e}-\mathbf{h}$ ). These data therefore confirmed the in vivo endogenous role of IL-33 in driving disease and tissue damage in CPT-11-induced intestinal mucositis.

\section{Neutrophil depletion attenuates CPT-11-induced intestinal mucositis}

We next investigated the effect of neutrophil depletion on CPT11 -induced intestinal mucositis. WT mice were treated with
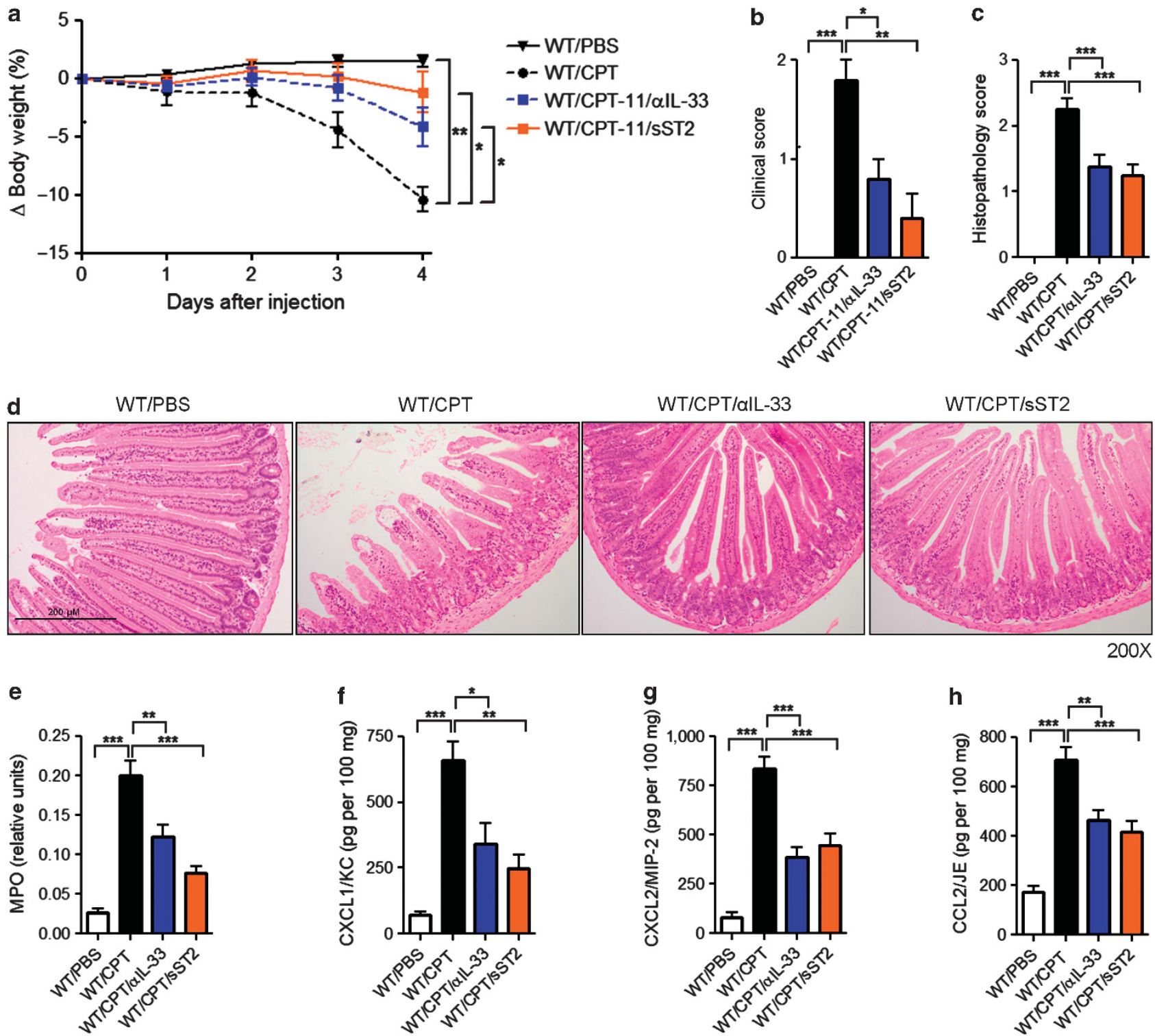

Figure 4 Interleukin-33 (IL-33) blockade ameliorates CPT-11-induced intestinal mucositis. Wild-type (WT) mice were treated with phosphate-buffered saline (PBS) or CPT-11 for 4 days and received anti-IL-33 (25 $\mu \mathrm{g}$ daily subcutaneously) or soluble ST2 (sST2; $100 \mu \mathrm{g}$ daily subcutaneously). Data were analyzed on day 4. (a) $\Delta$ Body weight loss, (b) clinical score, (c) histopathology score, and (d) representative histology (hematoxylin and eosin (H\&E) staining) of small intestine (SI), (e) Myeloperoxidase (MPO) activity of SI. (f) CXCL1/KC, (g) CXCL2/MIP-2, and (h) CCL2/JE production in the SI determined by enzyme-linked immunosorbent assay (ELISA). Results are representative of two independent experiments $(n=5$ mice per group). ${ }^{\star} P<0.05,{ }^{\star *} P<0.01$, and ${ }^{* \star *} P<0.001$. 
CPT-11 and then injected with anti-Ly6G antibody. The antibody effectively depleted neutrophils $\left(\mathrm{CD} 11 \mathrm{~b}^{+} \mathrm{CXCR}^{+}\right)$ in the SI as analyzed by fluorescence-activated cell sorting (up to $80 \%$, Supplementary Figure S2a). An intermediary dose of anti-Ly6G antibody ( $100 \mu \mathrm{g}$, every 2 days) was used so as to avoid development of early severe neutropenia in the present experimental setting. ${ }^{38}$ Neutrophil depletion significantly prevented the body weight loss and clinical disease in the CPT-11-treated and CPT-11- plus IL-33-treated WT mice compared with IgG-treated control mice (Figure 5a,b). The disease attenuation achieved by anti-Ly6G antibody was accompanied by a significant reduction of the SI histopathology in the antibody-treated mice (Figure 5c,d). Furthermore, neutrophil accumulation (Supplementary Figure S2b) and the
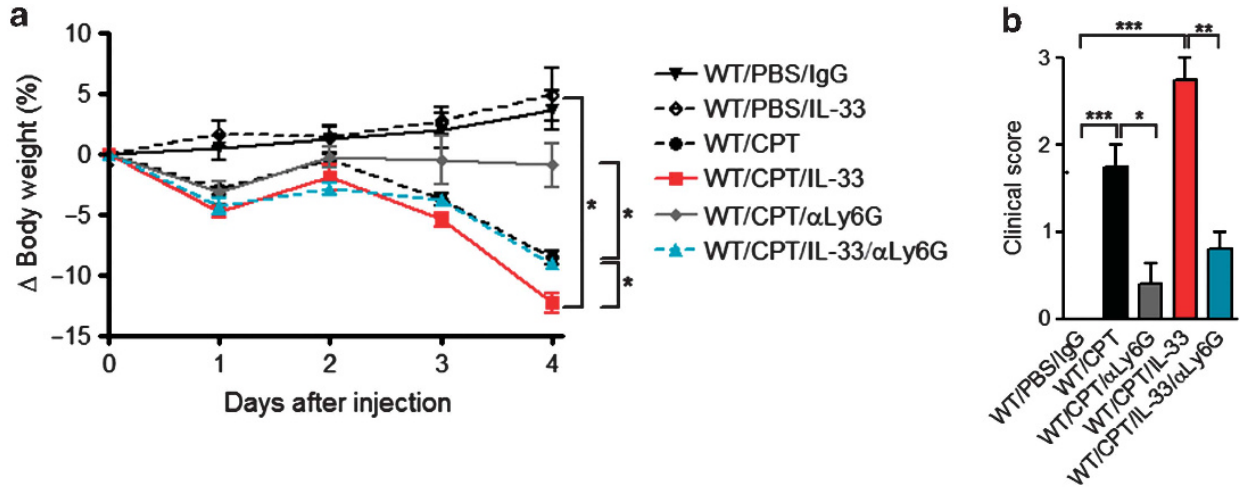

C
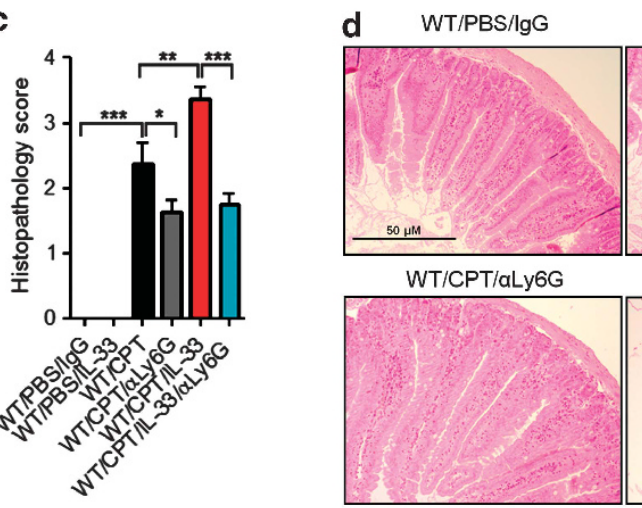

WT/PBS/LL-33
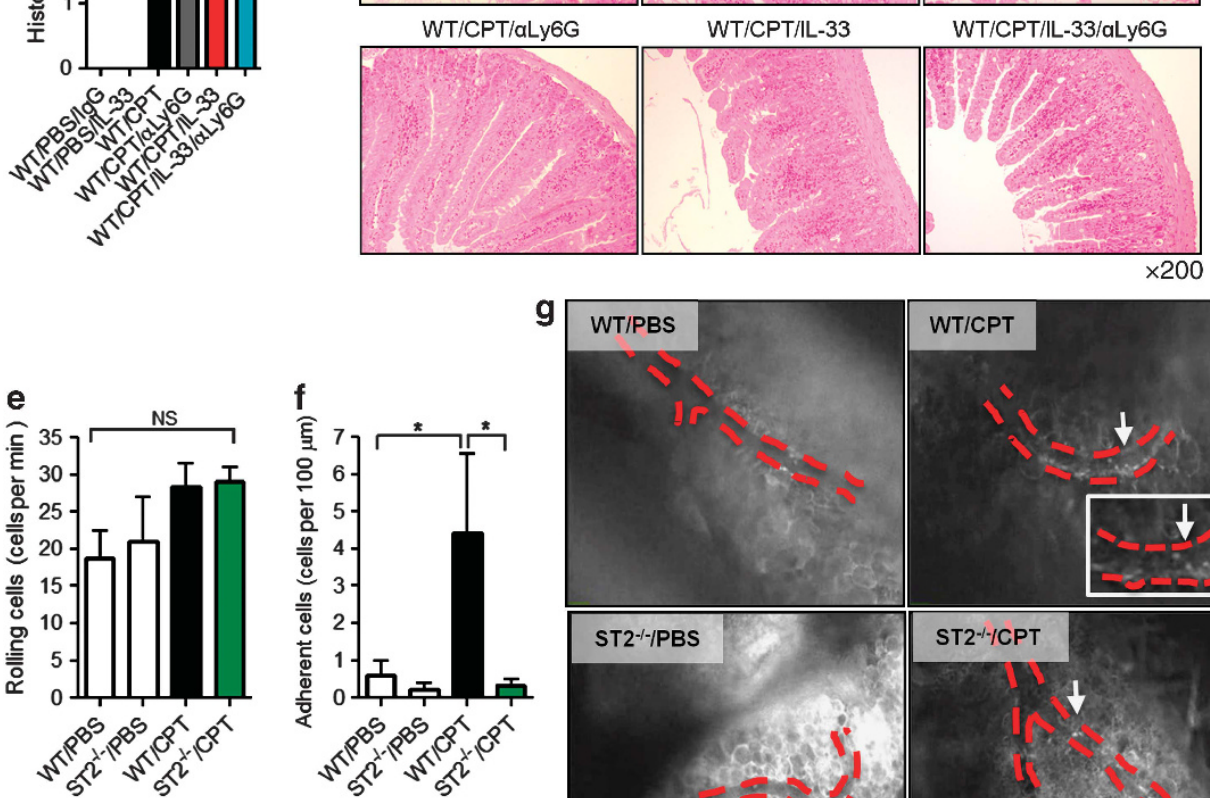

Figure 5 Neutrophil depletion attenuates CPT-11-induced intestinal mucositis. BALB/c mice were treated with CPT-11 with or without interleukin-33 (IL-33). Some mice were injected subcutaneously (s.c.) with anti-Ly6G (100 $\mu$ g) or control IgG on days 0 and 2. Data were analyzed on day 4. (a) $\Delta$ Body weight loss, (b) clinical score, (c) histopathology score, and (d) representative histology of small intestine (SI; hematoxylin and eosin (H\&E)). Intravital microscopy was used to assess the rolling (e) and adhesion (f) of leukocytes on the mesenteric microvasculature on day 2 in wild-type (WT) and ST2 ${ }^{-1-}$ mice. Data are pool of two experiments ( $n=8-10$ mice per group). (g) Representative pictures of the mesenteric veins. Red dotted lines, blood vessels; white arrows, leukocytes attached to the vessels. Results are representative of two independent experiments $\left(n=5\right.$ mice per group). ${ }^{*} P<0.05$, ${ }^{\star \star} P<0.01$, and ${ }^{\star \star *} P<0.001$. Also see Supplementary Videos online. 
production of CXCL1, CXCL2, CCL2, and IL-33 were also significantly reduced in the anti-Ly6G-treated mice (Supplementary Figure S2c-f). It may be that the accumulated activated neutrophils could cause further epithelial damage and facilitate the release of these chemokines and IL-33.

To visualize neutrophil migration, we performed intravital microscopy to assess the rolling and adhesion of leukocytes in the mesenteric microvasculature in $\mathrm{WT}$ and $\mathrm{ST} 2^{-1-}$ mice treated with CPT-11. No significant difference in the rolling of the leukocytes was observed between $\mathrm{WT}$ and ST2 ${ }^{-1-}$ mice (Figure 5e). In contrast, significantly more firm adhesion of leukocytes to the mesenteric endothelial veins was found in the WT mice treated with CPT-11 compared with untreated mice (Figure 5f). The increased cellular adhesion was not evident in the microvasculature of the ST2 $2^{-1-}$ mice (Figure 5f,g and Supplementary Video S1-S4). The leukocyte adhesion in the CPT-11-treated WT mice peaked on day 2 of treatment, coinciding with the onset of clinical signs and leukopenia.

CXCR2 is known to be an important receptor involved in neutrophil arrest in vivo. ${ }^{8}$ To further confirm the role of neutrophil migration in CPT-11-induced mucositis, we blocked the chemokine receptor CXCR2 in vivo using a specific antagonist, DF-2156A, that has been used to inhibit CXCR2 function on neutrophils and the migration of these cells. ${ }^{41}$ WT mice were treated with CPT-11 alone or with IL-33 as above. The mice were administered orally with a daily dose of DF-2156A. DF-2156A significantly reduced intestinal mucositis induced by CPT-11 and CPT-11 + IL-33 (Supplementary Figure S3a). DF-2156A also effectively blocked neutrophil infiltration in the SI (Supplementary Figure S3b). Furthermore, DF-2156A markedly reduced the production of CXCL1, CXCL2, and IL-33 by the SI of CPT-11-treated and CPT-11 + IL-33-treated mice (Supplementary Figure S3c-e). Significant reduction of the SI histopathology was also observed in DF2156A-treated mice compared with PBS-treated control mice (Supplementary Figure S3f and g). These results further indicate the critical role of neutrophil migration in the CPT-11induced mucositis.

Collectively, these data demonstrate that neutrophils play an important role in the onset and outcome of intestinal mucositis induced by CPT-11. Importantly, IL-33 participates in the cascade of events leading to neutrophil adhesion and accumulation in the SI during chemotherapy-induced mucositis.

\section{IL-33 targeting attenuates intestinal mucositis and extends effective tumor chemotherapy}

The important role of IL-33 in CPT-11-induced mucositis prompted us to investigate whether IL-33 blockade would enable a longer period of treatment with this cytotoxic drug against tumor, using a murine model of ectopic colon carcinoma induced by CT26 cells. WT and ST2 ${ }^{-1-}$ mice were injected subcutaneously (s.c.) with CT26 cells and CPT-11 treatment began $48 \mathrm{~h}$ later when the transplanted tumor was visible and/or palpable. The schedule of CPT-11 treatment was set to the minimum dose ( $45 \mathrm{mg} \mathrm{kg}^{-1}$ for 5 days) that is able to reduce CT26 tumor growth without being lethal to WT mice for at last 21 days from the start of treatment. The dose was reduced by half ( $22.5 \mathrm{mg} \mathrm{kg}^{-1}$ for 5 days) when the WT mice presented signs of mucositis; and the dose was reduced to half again (11.5 $\mathrm{mg} \mathrm{kg}^{-1}$ ) until days 18-21 after CT26 transplant. There was no significant difference in the rate of tumor growth and mortality between WT and ST2 ${ }^{-1-}$ mice without CPT-11 treatment (Figure 6a and Supplementary Figure S4a). WT mice treated with CPT-11 showed significant reduction in tumor size and survival. However, these mice developed severe intestinal mucositis and the treatment was withdrawn on day 5 . In contrast, ST2 ${ }^{-1-}$ showed no obvious sign of mucositis and continued to receive tapering doses of CPT- 11 . This prolonged continuous CPT-11 treatment resulted in further reduction of tumor size compared with that of CPT-11-treated WT mice. The beneficial outcome of CPT- 11 treatment in the ST2 ${ }^{-1-}$ mice was reflected in the significantly milder clinical score and reduced weight loss in these mice compared with the WT mice (Figure 6a and Supplementary Figure S4a).

We next investigated the effect of IL-33 blockade with antiIL-33 antibody. BALB/c mice were transplanted with CT26 and treated with CPT-11 as above. Some mice also received a daily i.p. injection of anti-IL-33 or IgG isotype control from days 2 to 12. As expected, mice given CPT-11 alone showed significantly reduced tumor size but severe mucositis. The treatment was withdrawn on day 6 . In contrast, mice administered with antiIL-33 developed mild mucositis and the continuous treatment with CPT-11 resulted in significantly further reduction in tumor size compared with mice given IgG control (Figure 6b and Supplementary Figure S4b). The beneficial outcome of anti-IL-33 injection was also evident in the reduced clinical score and body weight loss (Figure $\mathbf{6 b}$ ).

To confirm the beneficial effect of IL-33 blockade, we also treated mice with sST2. WT mice were injected with CPT- 11 as above and then received a daily i.p. injection of sST2 from days 2 to 12 . Mice given SST2 developed little or no mucositis and were able to receive continuous CPT-11 treatment. This treatment resulted in significant further reduction of tumor size compared with the control mice given PBS (Figure $\mathbf{6 c}$ and Supplementary Figure S4c). The relative absence of mucositis in the sST2-treated mice was also reflected in the reduced clinical score (reduced diarrhea) and body weight loss compared with PBS-treated mice (Figure 6c).

Collectively, these data demonstrate that IL-33 blockade effectively reduces mucositis and enables prolonged CPT-11 treatment, leading to a significant beneficial outcome of chemotherapy.

\section{DISCUSSION}

We demonstrate here that IL-33 mediates CPT-11-induced mucositis through the local production of CXCL1 and CXCL2 that recruits neutrophils to the site of inflammation causing tissue damage in the SI (Figure 7). Furthermore, blockade of the IL-33/ST2 pathway or neutrophil recruitment confers protection and tissue preservation. Importantly, IL-33 neutralization enables extended and effective chemotherapy in CT26 tumor-bearing mice, suggesting that the IL-33/ST2 pathway is a 

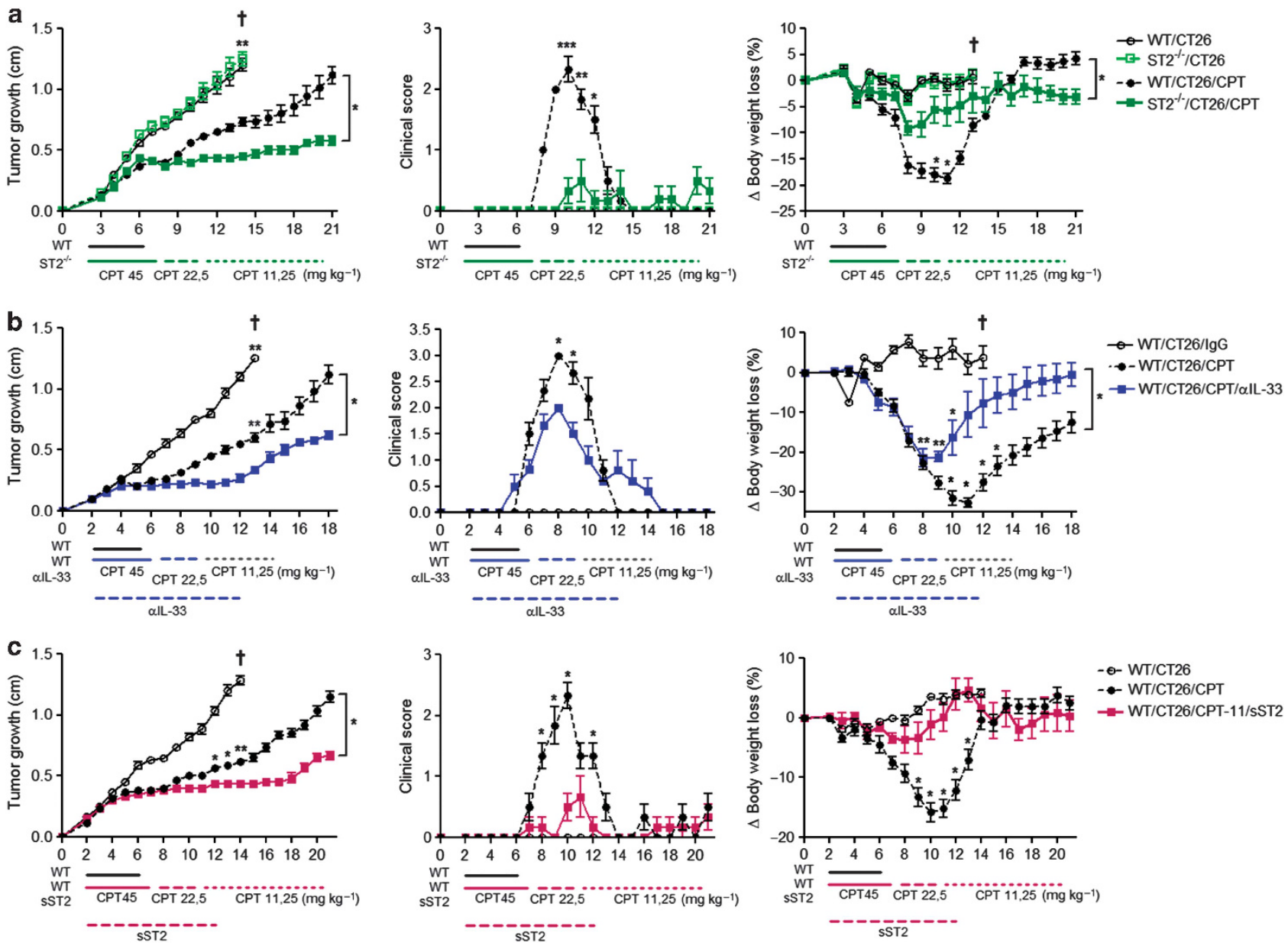

Figure 6 Interleukin-33 (IL-33) targeting attenuates intestinal mucositis and prolongs effective chemotherapy against tumor. Wild-type (WT) and $\mathrm{ST}^{-1-}$ mice were transplanted subcutaneously (s.c.) with CT26 colon carcinoma cells $\left(1 \times 10^{6}\right.$ cells). Some mice were treated with CPT-11 at indicated doses. Some WT mice were also injected daily s.c. (day 2-12) with anti-IL-33 (25 $\mu \mathrm{g})$ or soluble ST2 (sST2; $100 \mu \mathrm{g})$. Tumor growth (mean diameter in $\mathrm{cm}$ ), clinical score, and body weight loss were recorded daily. (a) WT and ST2 ${ }^{-}{ }^{-}$mice, (b) WT and anti-IL-33-treated mice, and (c) WT and sST2-treated mice. Results are representative of two independent experiments ( $n=6$ mice per group). ${ }^{\dagger}$ Mice were culled because of severity of disease. ${ }^{\star} P<0.05,{ }^{*} P<0.01$, and ${ }^{* * *} P<0.001$. See Supplementary Figure $\mathbf{S} 4$ online for pictures of tumor-bearing mice.

novel therapeutic target to alleviate mucosal damage leading to a beneficial outcome of cancer chemotherapy.

IL-33 is rapidly produced by SI epithelial cells (epTs) in the villi and crypts following exposure to CPT-11 in vivo and in vitro. IL-33 functions as a prototypic "alarmin," released upon cellular damage, stress, and necrosis, and may serve as a danger signal to alert the host of a local imbalance, such as trauma or infection. ${ }^{21,42}$ It is likely that both inactivation and release of IL-33 take place linking between apoptosis and cell damage in many acute and chronic inflammatory diseases in which IL-33 has been detected. Gut mucosal expression of IL33 is primarily localized to nonhematopoietic cells, particularly intestinal epTs. ${ }^{25,43}$ In active UC, IL-33 is localized to, and potently expressed by, epTs. ${ }^{24,43}$ Moreover, IL-33 has been shown to impair intestinal barrier function, where enhanced IL-33 may favor microbial translocation that perpetuates colonic inflammation in a model of dextran sodium sulfateinduced colitis in mice. ${ }^{23}$ We show here that CPT-11-induced
IL-33 led to markedly increased bacteremia in an ST2dependent manner. Together with the impaired intestinal barrier function, diarrhea in CPT-11-treated mice is thought to be caused by villous atrophy following crypt damage and apoptosis of absorptive cells in the SI. ${ }^{33,34}$ As diarrhea is one of the main drawbacks for cancer patients undergoing chemotherapy, ${ }^{32}$ attenuation of IL-33/ST2-dependant mucositis might also represent a therapeutic approach to diarrhea associated with CPT-11 treatment.

We have previously shown that IL-33 contributes to the increased expression of CXCR2 on neutrophils through the upregulation of GRK2 (G Protein-Coupled Receptor Kinase 2) following TLR4 activation in a model of polymicrobial sepsis. ${ }^{44}$ However, we did not observe any alteration in the expression of GRK2 on neutrophils harvested from the SI or blood following CPT-11+ IL-33 treatment (data not shown). This is perhaps not unexpected as, unlike sepsis, CPT-11-treatment induces neutrophil migration in the absence of massive infection. 


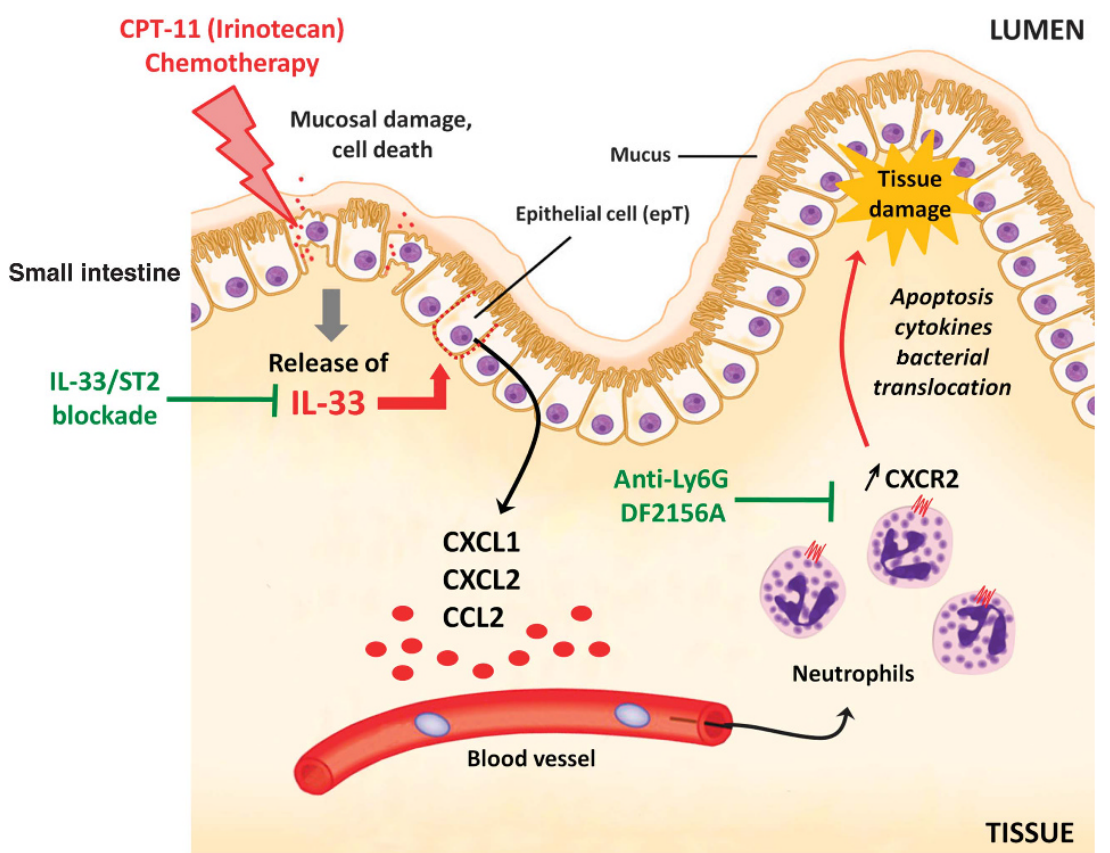

Figure 7 Schematic representation of the mechanism of interleukin-33 (IL-33)-mediated CPT-11-induced intestinal mucositis and potential therapeutic targets. CPT-11 damages the intestinal mucosa and releases IL-33 that enhances chemokine production from the epithelial cells and upregulation of CXCR2 on neutrophils, thereby increasing the recruitment of neutrophils to the inflammatory sites, leading to further tissue damage and bacterial translocation. Blocking of IL-33 by anti-IL-33 antibody or soluble ST2 (sST2) attenuates CXC chemokine production and neutrophil activation/ accumulation. Depletion of neutrophils or blocking CXCR2 would also attenuate tissue damage and disease outcome.

Bacteremia is related to epithelial damage late in the chemotherapy. ${ }^{9}$ The role of neutrophils in chemotherapyinduced mucosal damage was hitherto unknown. ${ }^{1,2}$ We demonstrate here that neutrophil depletion and CXCR2 blockade represent an efficient strategy to reduce the mucosal injury following epT release of IL-33 and proinflammatory cytokines.

Local production of IL-33 may induce a rapid release of proinflammatory mediators such as IL- $1 \beta$, IL-6, tumor necrosis factor- $\alpha$, and chemokines by a variety of innate immune cells including neutrophils, macrophages, and mast cells. ${ }^{16,22,45}$ During active UC and SAMP enteritis (using SAMP1/YitFc mice that develop spontaneous ileitis similar in many features to human Crohn's disease), IL-33 is markedly upregulated in colonic epTs and similar gut mucosal cells also express the receptor ST2, suggesting that IL-33 may act in an autocrine/ paracrine manner to amplify the inflammatory response in the context of SI inflammation. ${ }^{25}$ ST2 is present on the surface of neutrophils, although IL-33 is not directly involved in their recruitment. ${ }^{44}$ However, the robust production of the chemokines CXCL1 and CXCL2 indicates that IL-33 strongly contributes to the recruitment of neutrophils to the site of injury. Thus, strategies targeting the IL-33/CXC/neutrophil axis may help to limit collateral damage during responses to sterile injury by allowing neutrophils to remain intravascular as they navigate through healthy tissue to sites of injury. ${ }^{46}$ Even if blood neutropenia might compromise the host immune response at later time points, the early accumulation of neutrophils in the SI mucosa is a key event in driving tissue damage and disease during cancer chemotherapy. It should be noted that treatment with recombinant murine IL-33 alone without CPT-11 did not lead to significant pathology. It is likely that epithelial damage induced by the cytotoxic effect of CPT11 is necessary for the infiltration of neutrophil migration from the circulation to the inflammatory site. Alternatively, IL-33 may have to synergize with other mediators released by CPT11 -activated epTs to cause the damage in the SI. These potential accessory mediators remain to be defined.

Although many studies have addressed different strategies to enhance the effectiveness of CPT-11 and other compounds in reducing tumor growth, little attempt was made to alleviate mucosal damage and improve the duration and efficiency of the chemotherapy. ${ }^{3,6,14,33,34,47}$ Jovanovic et al. ${ }^{48,49}$ reported a modest reduction in tumor growth in a model of $4 \mathrm{~T} 1$ mammary carcinoma in ST2 ${ }^{-1-}$ mice as compared with WT mice, suggesting that IL-33 may contribute to tumor growth. ${ }^{48}$ We did not observe a significant difference in tumor growth between CT26-bearing WT and ST2 ${ }^{-1-}$ mice (Figure 6). This discrepancy could be because of the different types of tumors used.

The sST2 treatment appeared to be more effective than antiIL-33 in reducing mucositis, even though both treatments conferred significant protective effects in WT mice. However, this difference could be because of the suboptimal dose of antiIL-33 antibody used ( $25 \mu \mathrm{g}$ per mouse). The therapeutic basis and potential for the IL-33/ST2 blockade reside in the fact that 
IL-33 is released by epithelial cells as an "alarmin" in response to injury, whereas other cells in the epithelia may produce IL-33 in chronic IBD patients. ${ }^{22,23,25,50}$ Recent data of adult and pediatric UC and Crohn's disease patients demonstrate that specific IL-33 and ST2 gene polymorphisms confer an increased risk of developing IBD (both UC and Crohn's disease), reinforcing the involvement of the IL-33/ST2 axis in the onset of intestinal inflammation. ${ }^{51}$

In summary, we report here a pivotal role of IL-33 in mediating mucosal damage during cancer chemotherapy and that IL-33 blockade leads to a beneficial outcome of chemotherapy in a murine model of carcinoma. Importantly, IL-33 is found in considerable levels in the mucosa of IBD patients. ${ }^{24,52}$ Our results therefore suggest that inhibition of the IL-33/ST2 pathway may represent a novel approach to limit inflammatory mucosal damage and thus improve the effectiveness of chemotherapy.

\section{METHODS}

Animals. $\mathrm{BALB} / \mathrm{c}$ mice were purchased from Harlan-Olac, Bicester, UK. BALB/c St2 ${ }^{-1-}$ mice ${ }^{53}$ were bred and maintained at the University of Glasgow (Glasgow, UK). All experiments were performed in accordance with the UK Home Office guidelines. Mice under procedure were kept in polyethylene boxes with free access to soft food and water, and subjected to $12 \mathrm{~h}$ light-dark cycles.

Reagents and antibodies. Irinotecan hydrochloride (CPT-11, Camptosar) and SN-38 (7-Ethyl-10-hydroxycamptothecin) were purchased from Sigma-Aldrich (Poole, UK). Recombinant murine IL33 was obtained from Biolegend (Cambridge, UK). Mouse IL-33 monoclonal antibody (mAb; clone 396118) and isotype control were purchased from R\&D Systems (Abingdon, UK). Purified Rat antimouse Ly6G (clone 1A8) and isotype control were purchased from BD Biosciences (Oxford, UK). Recombinant murine sST2 was generated and purified from HEK293T cells at VIB Protein Service Facility, VIB Inflammation Research Center (Ghent, Belgium). DF-2156A was obtained from Dompé S.p.A. (L'Aquila, Italy).

Cell line. The human enterocyte-like cell line Caco-2 (ATCC HTB-37) and the CT26 (colon carcinoma from BALB/c, CT26.WT, ATCC CRL2638) were obtained from ATCC (LGC Standards, Middlesex, UK) and maintained in high-glucose Dulbecco's modified Eagle's medium (Gibco-BRL, Paisley, UK; Life Technologies, Paisley, UK) supplemented with $10 \%$ fetal calf serum (FCS) (LONZA, Slough, UK), $1 \%$ (vol/vol) HEPES (Gibco-BRL), 1\% (vol/vol) penicillin/streptomycin (Gibco-BRL), and $2 \mathrm{~mm}$ L-glutamine (Gibco-BRL). All cell cultures were carried out in $5 \% \mathrm{CO}_{2}$ in a humidified atmosphere at $37^{\circ} \mathrm{C}$. Cells were seeded at high density in T75 tissue culture flasks (Corning, Surrey, UK) and grown to $90 \%$ confluence. Before each experiment, culture medium and dead cells were aspirated off, and live cells were washed $3 \times$ with FCS-free culture medium. Cells were detached using $0.5 \%$ Trypsin-EDTA (Sigma-Aldrich), counted in Trypan blue dye, and used as indicated. Caco-2 cell monolayers were cultured for $48 \mathrm{~h}$ (100\% confluence) and incubated with CPT-11 (Sigma-Aldrich) or SN-38 (Sigma-Aldrich) dissolved in dimethyl sulfoxide (DMSO; Sigma-Aldrich) and then in supplemented medium (final DMSO concentration $\leq 0.2 \%$ ) using 24 -well culture plates (Corning) at $1 \times 10^{6}$ cells per ml. CT26 cells were washed twice in Hanks' balanced salt solution (HBSS, Sigma-Aldrich) to remove FCS contamination before s.c. injection in mice. Cell viability was verified by the MTT assay (Sigma-Aldrich). Briefly, cells were incubated with $300 \mu \mathrm{l}$ MTTcontaining RPMI medium ( $0.1 \mathrm{mg} \mathrm{ml}{ }^{-1}$ MTT in serum-free medium) for $4 \mathrm{~h}$. Medium was removed, and the formazan crystal formed in living cells was dissolved in $100 \mu \mathrm{l}$ DMSO per well. The relative viability (\%) was calculated based on absorbance at $550 \mathrm{~nm}$ using a microplate reader. Viability of nontreated control cells was arbitrarily defined as $100 \%$.

Induction of experimental intestinal mucositis. Experimental intestinal mucositis in mice was based on a model previously described by Ikuno et al. ${ }^{34}$ and Melo et al. ${ }^{10}$ with some modifications. Briefly, vehicle ( $45 \mathrm{mg} \mathrm{ml}^{-1}$ of sorbitol NF powder, $0.9 \mathrm{mg}$ of lactic acid, USP, and Dulbecco's phosphate-buffered saline (DPBS), $\mathrm{pH}$ 3.5-3.8) or CPT-11 (60 $\left.\mathrm{mg} \mathrm{kg}^{-1}\right)$ was administered i.p. for 2 (mild mucositis) or 4 (severe mucositis) consecutive days (from day 0 to day 3$).{ }^{54}$ Some mice were also treated i.p. with IL-33 (200 ng, 30 min before each CPT-11 injection), anti-IL-33 (25 $\mu$ g, daily, s.c.), anti-Ly6G (100 $\mu$ g, every 2 days, s.c.), sST2 (100 $\mu$ g, daily, s.c.), DF-2156A (10 $\mathrm{mg} \mathrm{kg}^{-1}$, orally daily), $\mathrm{PBS}$, or filtered water. On days 2 or 4 , mice were culled in a $\mathrm{CO}_{2}$ inhalation chamber. Blood was taken from the cava vein for leukocyte counts, DNA purification (sterile tubes), or serum preparation in heparinized tubes. SI was perfused, dissected, washed, measured, and stored at $-20^{\circ} \mathrm{C}$ for cytokine measurement by ELISA or at $-80^{\circ} \mathrm{C}$ for qPCR analysis. In some experiments, SI was used for histopathological analysis or digested for fluorescence-activated cell sorting analysis.

CT26 tumor induction. Mice were injected s.c. in the shaved right flank with CT26 cells at $1 \times 10^{6} / 50 \mu$ l. When the tumor had grown to $0.1-0.2 \mathrm{~cm}$ (palpable tumors, routinely on day 2 ), mice were injected i.p. with $200 \mu \mathrm{l}$ of CPT-11 (45 $\mathrm{mg} \mathrm{kg}^{-1}$ daily). The dose of CPT-11 was reduced to half of the initial dose for mice that had lost $\sim 15 \%$ of body weight. The size of tumor was monitored daily with a caliper. The tumor size was calculated and expressed as the average tumor diameters in $\mathrm{cm} \pm$ s.e.m. Mice were culled when appeared moribund or the tumor size reached $1.2 \mathrm{~cm}$ diameter.

Clinical score. The severity of diarrhea and body weight loss was monitored throughout the experimental periods. The severity of the diarrhea was scored as described by Kurita et al. ${ }^{47}$ as follows: 0 , normal, normal stool or absent; 1 , slight, slightly wet and soft stool; 2 , moderate, wet, and unformed stool with perianal staining of the coat; and 3, severe, watery stool with perianal staining of the coat.

Histopathological analysis. The SI was perfused, dissected, washed in HBSS, fixed in 4\% (v/v) neutral-buffered formalin (Merck, Merck Biosciences, Nottingham, UK), dehydrated, and embedded in paraffin. The tissues were cut into $5 \mu \mathrm{m}$ sections, stained with hematoxylineosin or periodic acid-Schiff, and examined with a Nikon Eclipse E400 microscope at $\times 200$ or $\times 400$ magnification (Louisville, KY). The severity of SI inflammation (leukocyte infiltration and tissue damage in the intestinal parenchyma) was assessed by a semiquantitative score (0-4) in a blinded manner. The severity of mucositis was graded using the following criteria as previously described: ${ }^{55}$ grade 0 , no lesion; grade $1,<10 \%$ crypts contain individual necrotic cells; grade $2,>10 \%$ crypts contain necrotic cells but the crypt architecture is intact; grade $3,>10 \%$ crypts contain necrotic cells showing focal loss of crypt architecture $(<20 \%)$, villi are shortened, and variable hypertrophy/hyperbasophilia apparent in the remaining crypt cells; grade 4, same as grade 3 except that the loss of crypt architecture and villous shortening are more extensive. Intestinal villus and crypt lengths were measured by an optical scale under $\times 200$ magnification, and villus-to-crypt length ratios calculated. Goblet cells were counted per hemi crypt/villus axis. A total of 25 crypts or hemi crypt/villus axis was counted in at least five different SI slides per experimental group.

Immunohistochemistry. The IHC demonstration of IL-33 or MPO protein in the SI was performed on paraffin sections of formalin-fixed samples using a mouse anti-IL-33 mAb (clone 396118), rat IgG2A, anti-Annexin V polyclonal antibody (Abcam, Cambridge, UK, ab14196), rabbit IgG, and human/mouse MPO Affinity Purified polyclonal $\mathrm{Ab}$, and goat IgG (all from R\&D Systems (Abingdon, UK) 
unless otherwise specified). Deparaffinized sections were incubated with anti-IL-33, anti-Annexin V, or anti-MPO antibody (diluted $1: 600-1: 1,000$ in $0.2 \%$ bovine serum albumin and 1:25 dilution of mouse serum) for $90 \mathrm{~min}$ at room temperature. The bound antibody was visualized by an avidin-biotin-peroxidase method (Vectastain Elite kit, Vector Laboratories, Burlingame, CA) and developed with 3,3'-diaminobenzidine (Sigma-Aldrich) in the presence of $\mathrm{H}_{2} \mathrm{O}_{2}$ as the chromogen. SI samples served as negative controls by omission of the primary antibody during otherwise identical incubations. SI samples from different control groups served as a further internal control. Staining was assessed by light microscopy $(\times 200$ and $\times 400)$.

Vascular permeability and neutrophil accumulation. The SI was perfused, washed, and removed at the indicated time points. The extravasation of Evans Blue dye into the SI was used as an index of increased vascular permeability, as previously described. ${ }^{56}$ Results are presented as the amount of Evans Blue in $\mu \mathrm{g}$ per $100 \mathrm{mg}$ of tissue in $1 \mathrm{ml}$. The extent of neutrophil accumulation in the SI was measured by MPO activity as previously described. ${ }^{56}$ Results are expressed as relative units (optical density $492 \mathrm{~nm}$ ) and corrected for the activity of other peroxidases not inhibited by 3-amino-1,2,4-triazole.

Western blotting analysis. SI samples were homogenized in RIPA buffer (Thermo Scientific, Cramlington, UK) containing protease inhibitors (Roche, West Sussex, UK). Supernatants were used as total lysates. Protein concentrations were estimated by the BCA protein assay (Thermo Scientific Pierce, Cramlington, UK). Supernatants were then boiled in reducing sodium dodecyl sulfate sample buffer and $30 \mu \mathrm{g}$ of protein lysate per lane were run through NuPAGE Novex 4-12\% Bis-Tris Protein Gels (Life Technologies) and transferred to Hybond ECL membranes (GE Healthcare, Buckinghamshire, UK). Membranes were blocked for $1 \mathrm{~h}$ in 5\% non-fat dried milk in DPBS and incubated overnight with the appropriate primary antibody at $4{ }^{\circ} \mathrm{C}$. Membranes were then washed in DPBS/Tween-20 and incubated with the appropriate secondary antibody. Both primary and secondary antibodies were diluted in 5\% non-fat dried milk in DPBS. Detection was performed by ECL Western Blotting Detection Reagents (GE Healthcare). Antibodies against cleaved poly (ADPribose) polymerase (Asp214, $\mathrm{mAb})$, Caspase $9(\mathrm{mAb})$, and all secondary antibodies were obtained from Cell Signaling Technologies, Hitchin, UK. Caspase-3 (rabbit polyclonal) and Bax (mAb) antibodies were obtained from Santa Cruz Biotechnology (Santa Cruz, CA).

ELISA. The concentrations of IL-33, sST2, IL-8, CXCL1, CXCL2, and CCL2 were analyzed by ELISA (all reagents were from R\&D Systems) on sera, tissue, or cell culture supernatants according to the manufacturer's instructions. The sensitivity of the assay was $<20 \mathrm{pg} \mathrm{ml}^{-1}$.

Intravital microscopy. An intra-vital microscope (ECLIPSE 50i, Nikon, Louisville, $\mathrm{KY}$ ) with a $\times 20$ objective lens was used to examine the mesenteric microcirculation on day 2 after CPT-11 injections $\left(60 \mathrm{mg} \mathrm{kg}^{-1}\right)$ in selected postcapillary venules within $40-60 \mu \mathrm{m}$. A digital camera (DS-Qi1MC, Nikon) was used to project the images onto a computer monitor, and the images were recorded for playback analysis with an Imaging Software (Nikon, Kawasaki). To measure the leukocyte-endothelial cell interactions, the fluorescent marker Rhodamine 6G (Sigma-Aldrich) was injected in the caudal vein (in a single bolus of $0.15 \mathrm{mg} \mathrm{kg}^{-1}$ ) immediately before measurements. The flux of rolling cells was measured as the number of cells that passed by a given point per min. A leukocyte was considered to be adherent if it remained stationary for at least $30 \mathrm{~s}$. Total leukocyte adhesion was quantified as the number of adherent cells in the intravascular space within an area of $100 \mu \mathrm{m}$.

Isolation of epTs from the SI. SI from naive $B A L B / c$ mice was harvested, cut in $5 \mathrm{~cm}$-long pieces, and the luminal surface exposed. Samples were incubated in PBS-EDTA (30 mM) for $15 \mathrm{~min}$ at $37^{\circ} \mathrm{C}$ under mild agitation to remove the epithelial layer. Epithelial cell preparation was filtered through $70 \mu \mathrm{m}$ mesh, washed in RPMI-10\% FCS, and filtered again. Epithelial cells were counted and $2 \times 10^{6}$ cells were cultured in RPMI-10\% FCS for $6 \mathrm{~h}$ with CPT-11 or RPMI with $0.1 \%$ DMSO as vehicle control.

Preparation and culture of SI explants. SI was opened longitudinally and washed thoroughly in sterile DPBS supplemented with 2\% FCS and $1 \%$ penicillin/streptomycin. Fat tissue was removed and segments with peyer patches were excluded. Three to five segments from terminal duodenum of $1 \mathrm{~cm}$ in length per mice were placed in 24-well culture plates (Corning) containing fresh RPMI 1640 (Life Technologies) supplemented with $10 \%$ FCS and $1 \%$ penicillin/streptomycin, and incubated at $37^{\circ} \mathrm{C}$ for $24 \mathrm{~h}$. Segments were kept with the luminal surface exposed to the medium. Reagents of interest were added to the culture for $24 \mathrm{~h}$. DMSO concentration was used at $\leq 0.2 \%$. Supernatants were then harvested, centrifuged at $13,000 \times g$, and stored at $-20^{\circ} \mathrm{C}$ until used. Some explants were preincubated with $10 \mu \mathrm{g} \mathrm{ml}^{-1} \mathrm{sST} 2$ for $40 \mathrm{~min}$ at $37^{\circ} \mathrm{C}$.

Neutrophil isolation and chemotaxis assay. Neutrophils were purified from BALB/c bone marrow by positive selection using a Ly6G isolation kit (Miltenyi Biotechnology, Bisley, UK). Ly6G ${ }^{+}$cells were resuspended in chemotaxis buffer $(0.5 \%$ bovine serum albumin in RPMI-1640) and neutrophil migration was assayed in 24-well sterile polystyrene plates with transwell permeable supports containing $5 \mu \mathrm{m}$ pore size polycarbonate membranes (Corning). The mCXCL2 (10 $\mathrm{ng} \mathrm{ml}^{-1}$, Peprotech) that was able to induce the recruitment of $1 \times 10^{5}$ purified ${\text { Ly } 6 \mathrm{G}^{+}}^{+}$neutrophils per $\mathrm{ml}$ to the lower chamber after $3 \mathrm{~h}$ was used as positive control (data not shown). Freshly collected supernatants from SI explants were added in the lower chamber $(600 \mu \mathrm{l}$ per well). As negative control, chemotaxis buffer (bovine serum albumin + RPMI) only was added. Neutrophils $\left(1 \times 10^{6} / 100 \mu \mathrm{l}\right)$ were added in the top chamber. The plate was then incubated for $3 \mathrm{~h}$ at $37^{\circ} \mathrm{C}$ in $5 \% \mathrm{CO}_{2}$. Migrated neutrophils were recovered from the lower chamber, centrifuged, resuspended, and counted under light microscopy $(\times 400)$ using a Neubauer hemocytometer.

Isolation of SI leukocytes. The SI of mice were perfused, removed, and washed in HBSS. Fat tissue and peyer patches were removed; the SI was opened longitudinally, washed in 2\% FCS in HBSS, and cut into $0.5 \mathrm{~cm}$ sections. Tissue was then placed in $10 \mathrm{ml} \mathrm{HBSS} / 2 \%$ FCS and shaken for $15 \mathrm{~min}$ at $37^{\circ} \mathrm{C}$, and the supernatant discarded. Freshly prepared calcium/magnesium-free HBSS ( $10 \mathrm{ml}$, Life Technologies) containing 2 mm EDTA (Sigma-Aldrich) was then added. The tube was placed in a shaking incubator for $15 \mathrm{~min}$ at $37^{\circ} \mathrm{C}$, and the supernatant discarded. Calcium/magnesium-free HBSS $(10 \mathrm{ml})$ was then added, the tube was shaken, and the supernatant discarded. This step was repeated, and the remaining tissue was digested with $1.25 \mathrm{mg} \mathrm{ml}^{-1}$ collagenase D (Roche Diagnostic Systems, Mannheim, Germany), $1 \mathrm{mg} \mathrm{ml}^{-1}$ Dispase (Life Technologies), and $0.5 \mathrm{mg} \mathrm{ml}^{-1}$ DNase (Sigma-Aldrich) in complete RPMI-1640 for 30-40 min in a shaker at $37^{\circ} \mathrm{C}$ until complete digestion of the tissue. The final supernatant was passed twice through $100 \mu \mathrm{m}$ cell strainers (Falcon, Oxford, UK). Cells were centrifuged, washed, and counted in Trypan blue.

Flow cytometry. Leukocytes $\left(\sim 1 \times 10^{6}\right.$ cells/tube) were stained with 4',6-diamidino-2-phenylindole (cell viability), anti-CD45-Pacific Blue, anti-F4/80-FITC, anti-CD11b-PercP Cy5.5, anti-Ly6G-APC, anti-CXCR2-PE (all from R\&D Systems), anti-CD62L-FITC, antiGRK2-FITC, anti-Cytokeratin (C-11)-FITC (Abcam), anti-IL-33-PE (R\&D Systems), and isotype controls (BD Biosciences), following the manufacturer's protocols. IL-33 production by CD $45^{-}$Cytokeratin ${ }^{+}$ epT cells was determined after stimulation with phorbol 12-myristate 13 -acetate $\left(50 \mathrm{ng} \mathrm{ml}^{-1}\right)$ and ionomycin $\left(1 \mu \mathrm{g} \mathrm{ml}^{-1}\right)$ in the presence of GolgiStop and permeabilization of the cells (Cytofix/Cytoperm, BD Biosciences). Cells were acquired using a Beckman Coulter CyAn ADP (Beckman Coulter, High Wycombe, UK). Gating strategy 
(Supplementary Figure S5) and analysis were performed using the FlowJo software (TreeStar Software, Ashland, OR).

Real-time PCR. For RNA extraction, SI was isolated and preserved in RNAlater (Qiagen, Crawley, UK). The tissue was homogenized in TRIzol reagent (Sigma-Aldrich) and RNA was extracted according to standard protocol (RNeasy kit, Qiagen). For quantitative real-time PCR analysis, complementary DNA was amplified using M-MLV Reverse Transcriptase (Promega, Southampton, UK). Relative quantities of mRNA was determined using SYBR Green PCR Master Mix (Applied Biosystems) and by the comparative threshold cycle method provided by the manufacturer for the ABI Prism 7700/ 7900 HT Sequence Detection System. Primers were as follows: mouse bax, Forward $5^{\prime}$-TTGCTGATGGCAACTTCAACTGGG- $3^{\prime}$ and Reverse $5^{\prime}$-TGTCCAGCCCATGATGGTTCTGAT-3'. Mouse casp3, Forward $5^{\prime}$-TGGCAACGGAATTCGAGTCCTTCT-3' and Reverse 5'-TGAGCATGGACACAATACACGGGA-3'. Relative expression levels were calculated as $\Delta \mathrm{Ct}$ values by normalizing $\mathrm{Ct}$ values of target genes to $\mathrm{Ct}$ values of hypoxanthine phosphoribosyl transferase-1 (hprt1). Data are presented as relative \% of expression. For the detection of Eubacteria, blood samples were collected from mice on day 4 using sterile material (endotoxin free/pyrogen free, Costar, High Wycombe, UK) and total DNA extracted using the DNeasy Blood and Tissue Kit (Qiagen). A standard curve was prepared using purified DNA from Escherichia coli (concentration range of $1 \mathrm{pg}$ to $1 \mu \mathrm{g} \mathrm{ml}^{-1}$ ). The amount of Eubacteria DNA was determined using SYBR Green PCR Master Mix (Applied Biosystems) with the standard curve as a reference for the quantification of DNA per sample. Primers were as follows: UniF340, 5'-ACTCCTACGGGAGGCAGCAGT-3'; UniR514, 5' -ATTACCGCGGCTGCTGGC-3'.

Statistical analysis. Results are shown as means \pm s.e.m. Differences were compared by using one-way analysis of variance followed by Student-Newman-Keuls or Bonferroni post hoc analysis using the Graph Prism Software 4.0 (La Jolla, CA). Tumor size and body weight were analyzed using two-way analysis of variance. All data are representative of at least two experiments. Results with a $P<0.05$ were considered significant.

SUPPLEMENTARY MATERIAL is linked to the online version of the paper at http://www.nature.com/mi

\section{ACKNOWLEDGMENTS}

This work was supported by the Wellcome Trust and the Medical Research Council, UK (to F.Y.L. and G.J.G.); the Belgian Federation against Cancer, MRP-Group-ID, IWT-SBO, and FWO programs (to R.B.); CNPq, FAPEMIG, and CAPES, Brazil (to M.M.T. and G.B.M.); and the European Community's Seventh Framework Programme (FP7-2007-2013) under grant agreement no. HEALTH-F4-2011-281608 (to M.M.T. and G.J.G.). We thank Mr lain MacMillan (Veterinary School, University of Glasgow) for help with histology and $\mathrm{IHC}$.

\section{DISCLOSURE}

The authors declared no conflict of interest.

(c) 2014 Society for Mucosal Immunology

\section{REFERENCES}

1. Sonis, S.T. The pathobiology of mucositis. Nat. Rev. Cancer 4, 277-284 (2004).

2. Sonis, S.T. A biological approach to mucositis. J. Support Oncol. 2, 21-32 (2004). discussion 35-26.

3. Mathijssen, R.H. et al. Clinical pharmacokinetics and metabolism of irinotecan (CPT-11). Clin. Cancer Res. 7, 2182-2194 (2001).

4. Hebbar, M., Ychou, M. \& Ducreux, M. Current place of high-dose irinotecan chemotherapy in patients with metastatic colorectal cancer. J. Cancer Res. Clin. Oncol. 135, 749-752 (2009).
5. Paduch, R., Kandefer-Szerszen, M. \& Piersiak, T. The importance of release of proinflammatory cytokines, ROS, and $\mathrm{NO}$ in different stages of colon carcinoma growth and metastasis after treatment with cytotoxic drugs. Oncol. Res. 18, 419-436 (2010).

6. Kawato, Y., Aonuma, M., Hirota, Y., Kuga, H. \& Sato, K. Intracellular roles of $\mathrm{SN}-38$, a metabolite of the camptothecin derivative CPT-11, in the antitumor effect of CPT-11. Cancer Res. 51, 4187-4191 (1991).

7. Logan, R.M., Stringer, A.M., Bowen, J.M., Gibson, R.J., Sonis, S.T. \& Keefe, D.M. Serum levels of NFkappaB and pro-inflammatory cytokines following administration of mucotoxic drugs. Cancer Biol. Ther. 7, 1139-1145 (2008).

8. Logan, R.M., Stringer, A.M., Bowen, J.M., Gibson, R.J., Sonis, S.T. \& Keefe, D.M. Is the pathobiology of chemotherapy-induced alimentary tract mucositis influenced by the type of mucotoxic drug administered? Cancer Chemother. Pharmacol. 63, 239-251 (2009).

9. Leitao, R.F. et al. Role of inducible nitric oxide synthase pathway on methotrexate-induced intestinal mucositis in rodents. BMC Gastroenterol. 11, 90 (2011).

10. Melo, M.L. et al. Role of cytokines (TNF-alpha, IL-1beta and KC) in the pathogenesis of CPT-11-induced intestinal mucositis in mice: effect of pentoxifylline and thalidomide. Cancer Chemother. Pharmacol. 61, 775-784 (2008).

11. Vento, S. \& Cainelli, F. Infections in patients with cancer undergoing chemotherapy: aetiology, prevention, and treatment. Lancet Oncol. 4, 595-604 (2003).

12. Brandi, G. et al. Intestinal microflora and digestive toxicity of irinotecan in mice. Clin. Cancer Res. 12, 1299-1307 (2006).

13. Arcaroli, J.J. et al. ALDH + tumor-initiating cells exhibiting gain in $\mathrm{NOTCH} 1$ gene copy number have enhanced regrowth sensitivity to a gamma-secretase inhibitor and irinotecan in colorectal cancer. Mol. Oncol. 6, 370-381 (2012).

14. Finnberg, N. et al. Non-invasive fluorescence imaging of cell death in fresh human colon epithelia treated with 5-Fluorouracil, CPT-11 and/or TRAIL. Cancer Biol. Ther. 4, 937-942 (2005).

15. Schmitz, J. et al. IL-33, an interleukin-1-like cytokine that signals via the IL1 receptor-related protein ST2 and induces T helper type 2-associated cytokines. Immunity 23, 479-490 (2005).

16. Liew, F.Y., Pitman, N.I. \& Mclnnes, I.B. Disease-associated functions of IL-33: the new kid in the IL-1 family. Nat. Rev. Immunol. 10, 103-110 (2010).

17. Luthi, A.U. et al. Suppression of interleukin-33 bioactivity through proteolysis by apoptotic caspases. Immunity 31, 84-98 (2009).

18. Tominaga, S. A putative protein of a growth specific CDNA from BALB/c3T3 cells is highly similar to the extracellular portion of mouse interleukin 1 receptor. FEBS Lett. 258, 301-304 (1989).

19. Xu, D. et al. Selective expression of a stable cell surface molecule on type 2 but not type 1 helper T cells. J. Exp. Med. 187, 787-794 (1998).

20. Kumar, S., Tzimas, M.N., Griswold, D.E. \& Young, P.R. Expression of ST2, an interleukin-1 receptor homologue, is induced by proinflammatory stimuli. Biochem. Biophys. Res. Commun. 235, 474-478 (1997).

21. Cayrol, C. \& Girard, J.-P. The IL-1-like cytokine IL-33 is inactivated after maturation by caspase-1. Proc. Natl. Acad. Sci. USA 106, 9021-9026 (2009).

22. Pastorelli, L., De Salvo, C., Vecchi, M. \& Pizarro, T.T. The role of IL-33 in gut mucosal inflammation. Mediators Inflamm. 2013, 608187 (2013).

23. Sedhom, M.A. et al. Neutralisation of the interleukin-33/ST2 pathway ameliorates experimental colitis through enhancement of mucosal healing in mice. Gut 62, 1714-1723 (2012).

24. Beltran, C.J. et al. Characterization of the novel ST2/IL-33 system in patients with inflammatory bowel disease. Inflamm. Bowel Dis. 16, 1097-1107 (2010).

25. Pastorelli, L. et al. Epithelial-derived IL-33 and its receptor ST2 are dysregulated in ulcerative colitis and in experimental Th1/Th2 driven enteritis. Proc. Natl. Acad. Sci. USA 107, 8017-8022 (2010).

26. Seidelin, J.B., Rogler, G. \& Nielsen, O.H. A role for interleukin-33 in $T(H) 2-$ polarized intestinal inflammation?. Mucosal. Immunol 4, 496-502 (2011).

27. Pushparaj, P.N. et al. Interleukin-33 exacerbates acute colitis via interleukin-4 in mice. Immunology 140, 70-77 (2013).

28. Stringer, A.M. et al. Irinotecan-induced mucositis manifesting as diarrhoea corresponds with an amended intestinal flora and mucin profile. Int. J. Exp. Pathol. 90, 489-499 (2009). 
29. Stringer, A.M. et al. Irinotecan-induced mucositis is associated with changes in intestinal mucins. Cancer Chemother. Pharmacol. 64, 123-132 (2009).

30. Lima-Junior, R.C. et al. Involvement of nitric oxide on the pathogenesis of irinotecan-induced intestinal mucositis: role of cytokines on inducible nitric oxide synthase activation. Cancer Chemother. Pharmacol. 69, 931-942 (2012).

31. Sultani, M., Stringer, A.M., Bowen, J.M. \& Gibson, R.J. Anti-inflammatory cytokines: important immunoregulatory factors contributing to chemotherapy-induced gastrointestinal mucositis. Chemother. Res. Pract. 2012, 490804 (2012).

32. Stein, A., Voigt, W. \& Jordan, K. Chemotherapy-induced diarrhea: pathophysiology, frequency and guideline-based management. Ther. Adv. Med. Oncol. 2, 51-63 (2010).

33. Guffroy, M. \& Hodge, T. Re:Irinotecan (CPT-11) and characteristic mucosal changes in the mouse ileum and cecum. J. Natl. Cancer Inst. 88, 1240-1241 (1996).

34. Ikuno, N., Soda, H., Watanabe, M. \& Oka, M. Irinotecan (CPT-11) and characteristic mucosal changes in the mouse ileum and cecum. J. Natl. Cancer Inst. 87, 1876-1883 (1995).

35. Bodey, G.P. Antibiotic therapy of infections in patients undergoing cancer chemotherapy. Antibiot. Chemother. 18, 49-88 (1974).

36. Le, H., Kim, W., Kim, J., Cho, H.R. \& Kwon, B. Interleukin-33: a mediator of inflammation targeting hematopoietic stem and progenitor cells and their progenies. Front. Immunol. 4, 104 (2013).

37. Su, Z. et al. Potential autocrine regulation of interleukin-33/ST2 signaling of dendritic cells in allergic inflammation. Mucosal Immunol. 6, 921-930 (2013).

38. Blijlevens, N.M., Logan, R.M. \& Netea, M.G. The changing face of febrile neutropenia-from monotherapy to moulds to mucositis. Mucositis: from febrile neutropenia to febrile mucositis. J. Antimicrob. Chemother. 63 (Suppl 1), i36-i40 (2009).

39. Soares, P.M., Lopes, L.O., Mota, J.M., Belarmino-Filho, J.N., Ribeiro, R.A. \& Souza, M.H. Methotrexate-induced intestinal mucositis delays gastric emptying and gastrointestinal transit of liquids in awake rats. Arq. Gastroenterol. 48, 80-85 (2011).

40. Fijlstra, M., Rings, E.H., Verkade, H.J., van Dijk, T.H., Kamps, W.A. \& Tissing, W.J. Lactose maldigestion during methotrexate-induced gastrointestinal mucositis in a rat model. Am. J. Physiol. Gastrointest. Liver Physiol. 300, G283-G291 (2011).

41. Bertini, R. et al. Receptor binding mode and pharmacological characterization of a potent and selective dual CXCR1/CXCR2 non-competitive allosteric inhibitor. Br. J. Pharmacol. 165, 436-454 (2012).
42. Moussion, C., Ortega, N. \& Girard, J.P. The IL-1-like cytokine IL-33 is constitutively expressed in the nucleus of endothelial cells and epithelial cells in vivo: a novel "alarmin"? PLoS One 3, e3331 (2008).

43. Kobori, A. et al. Interleukin-33 expression is specifically enhanced in inflamed mucosa of ulcerative colitis. J. Gastroenterol. 45, 999-1007 (2010).

44. Alves-Filho, J.C. et al. Interleukin-33 attenuates sepsis by enhancing neutrophil influx to the site of infection. Nat. Med. 16, 708-712 (2010).

45. Oboki, K. et al. IL-33 is a crucial amplifier of innate rather than acquired immunity. Proc. Natl. Acad. Sci. USA 107, 18581-18586 (2010).

46. McDonald, B. et al. Intravascular danger signals guide neutrophils to sites of sterile inflammation. Science 330, 362-366 (2010).

47. Kurita, A., Kado, S., Kaneda, N., Onoue, M., Hashimoto, S. \& Yokokura, T. Alleviation of side effects induced by irinotecan hydrochloride (CPT-11) in rats by intravenous infusion. Cancer Chemother. Pharmacol. 52, 349-360 (2003).

48. Jovanovic, I. et al. ST2 deletion enhances innate and acquired immunity to murine mammary carcinoma. Eur. J. Immunol. 41, 1902-1912 (2011).

49. Milovanovic, M. et al. IL-33/ST2 axis in inflammation and immunopathology. Immunol. Res. 52, 89-99 (2012).

50. Salas, A. The IL-33/ST2 axis: yet another therapeutic target in inflammatory bowel disease? Gut 62, 1392-1393 (2013).

51. Latiano, A. et al. Associations between Genetic Polymorphisms in IL-33, IL1R1 and Risk for Inflammatory Bowel Disease. Plos One 8, e62144 (2013).

52. Seidelin, J.B., Bjerrum, J.T., Coskun, M., Widjaya, B., Vainer, B. \& Nielsen, O.H. IL-33 is upregulated in colonocytes of ulcerative colitis. Immunol. Lett. 128, 80-85 (2010).

53. Townsend, M.J., Fallon, P.G., Matthews, D.J., Jolin, H.E. \& McKenzie, A.N. T1/ST2-deficient mice demonstrate the importance of T1/ST2 in developing primary Thelper cell type 2 responses. J. Exp. Med. 191, 1069-1076 (2000).

54. Guichard, S., Chatelut, E., Lochon, I., Bugat, R., Mahjoubi, M. \& Canal, P. Comparison of the pharmacokinetics and efficacy of irinotecan after administration by the intravenous versus intraperitoneal route in mice. Cancer Chemother. Pharmacol. 42, 165-170 (1998).

55. Xiang, D. et al. Interleukin-1 receptor antagonist attenuates cyclophosphamide-induced mucositis in a murine model. Cancer Chemother. Pharmacol. 67, 1445-1453 (2011).

56. Souza, D.G., Guabiraba, R., Pinho, V., Bristow, A., Poole, S. \& Teixeira, M.M. IL-1-driven endogenous IL-10 production protects against the systemic and local acute inflammatory response following intestinal reperfusion injury. J. Immunol. 170, 4759-4766 (2003). 